\title{
Piezoelectric Wafer Active Sensor Embedded Ultrasonics in Beams and Plates
}

\author{
by V. Giurgiutiu. J. Bao and W. Zhao
}

\begin{abstract}
In this paper we present the results of a systematic theoretical and experimental investigation of the fundamental aspects of using piezoelectric wafer active sensors (PWASs) to achieve embedded ultrasonics in thin-gage beam and plate structures. This investigation opens the path for systematic application of PWASs for in situ health monitoring. After a comprehensive review of the literature, we present the principles of embedded PWASs and their interaction with the host structure. We give a brief review of the Lamb wave principles with emphasis on the understanding the particle motion wave speed/group velocity dispersion. Finite element modeling and experiments on thin-gage beam and plate specimens are presented and analyzed. The axial $\left(S_{0}\right)$ and flexural $\left(A_{0}\right)$ wave propagation patterns are simulated and experimentally measured. The group-velocity dispersion curves are validated. The use of the pulse-echo ultrasonic technique with embedded PWASs is illustrated using both finite element simulation and experiments. The importance of using highfrequency waves optimally tuned to the sensor-structure interaction is demonstrated. In conclusion, we discuss the extension of these results to in situ structural health monitoring using embedded ultrasonics.
\end{abstract}

KEY WORDS-Piezoelectric wafers, piezoelectric sensors, active sensors, in situ diagnostics, structural health monitoring, piezoelectrics, ultrasonics, elastic waves, P-waves, Swaves, shear waves, axial waves, flexural waves, Rayleigh waves, Lamb waves, plate waves, surface waves, wave speed, dispersion, group velocity, pulse-echo, acoustoultrasonics

\section{Introduction}

Piezoelectric wafer active sensor ultrasonics is a relatively new method for in situ non-destructive evaluation (NDE). Elastic waves propagating in material carry the information about defects. This information can be extracted by analyzing the wave signals. A large body of knowledge exists on using elastic waves for damage detection, either by ultrasonic (e.g., Krautkramer and Krautkramer, ${ }^{1}$ Blitz and Simpson, ${ }^{2}$ Cawley $^{3}$ and Rose ${ }^{4,5}$ ) or acousto-ultrasonic ${ }^{6}$ means. Due to the physical properties of wave propagation, large areas can be interrogated with a few transducers. This simplifies the process of detecting and characterizing defects. However, conventional ultrasonic transducers are expensive and rather

\footnotetext{
V. Giurgiutiu (victorg@sc.edu) (SEM member) is an Associate Professor, J. Bao is a former PhD student and W. Zhao is a Research Professor, Department of Mechanical Engineering, University of South Carolina, Columbia, SC 29208, USA.
}

Original manuscript submitted: August 6, 2001.

Final manuscript received: March 24, 2003. bulky devices, which incorporate the piezoelectric resonator (piezoelectric stack, proof mass, and damper), the casing, and the electric cord. Due to their cost and size, conventional ultrasonic devices are unlikely to be deployable in fixed sensor-array installations to achieve permanent structural health monitoring objectives. In their place, a new generation of sensors has emerged: piezoelectric wafer active sensors (PWASs). PWASs are small, inexpensive, unobtrusive, and non-invasive permanently attached piezoelectric wafers that can conceivably be deployed in health monitoring arrays without producing prohibitive weight and cost penalties.

Piezoelectric excitation of isotropic and anisotropic structures was initially studied for structural control. Crawley and Lazarus ${ }^{7}$ developed piezoelectric sensor and actuator relations using equivalent line moments. This work was extended by Tzou and Tseng, ${ }^{8}$ Dimitriadis et al., ${ }^{9}$ Lester and Lefebvre, ${ }^{10}$ Song and Librescu ${ }^{11}$ and others. Saravanos and Heyliger ${ }^{12}$ developed a unified approach for laminated beams with incorporated piezoelectric elements. Damage detection through built-in piezoelectrics was pioneered by Keilers and Chang ${ }^{13}$ for identification of delaminations in composite plates. Various wave propagation based damage detection methods have been recently studied. ${ }^{14,15}$ Moetakef et al. ${ }^{16}$ performed finite-element modeling and an experimental study of elastic wave generation through equally spaced piezoceramic wafers. Lakshmanan and Pines ${ }^{17}$ used wave propagation to detect transverse cracks in a rotating composite beam from the structural scattering properties. Blanas et al. ${ }^{18,19}$ studied the use of active composite sensors for acoustic-emission health monitoring. Lichtenwalner ${ }^{20}$ used piezoelectrics for local area damage detection. Jiang et al. ${ }^{21}$ used aluminum beams instrumented with piezoelectric wafer transducers to study the longitudinal wave propagation for structural health monitoring purposes. Osmont et al. ${ }^{22}$ used Lamb wave propagation in sandwich plates to identify the effect of impact damage in sandwich skins and core. Giurgiutiu et al. ${ }^{23}$ outlined the general methodology for using piezoelectric active sensors for health monitoring of aging aircraft structures.

The above-mentioned exploratory studies have shown that PWASs are a feasible instrumentation option for in situ damage detection and structural health monitoring using wave propagation techniques. However, this novel approach still lacks the maturity of conventional ultrasonics and acoustoultrasonic techniques, and the understanding of its underlying principles and limitations is still incomplete. None of the studies published so far has performed a systematic investigation of how the elastic waves are generated and received 
by the piezoelectric wafers, and how they interact with the defect. In addition, these studies were limited to low-frequency flexural Lamb waves in the $10-20 \mathrm{kHz}$ range. At this low frequency, the pulse-echo method cannot be applied, since the reflected echo superposed over the emerging wave pulse. In this paper we describe a systematic study aimed at furthering the understanding of this novel damage detection technique that resembles conventional ultrasonics and acoustoultrasonics but relies on a different type of structural excitation and different type of transducer. In this study, both theoretical and experimental techniques were used. In order to keep the problem tractable, simple geometries (beam and plates) were used. In addition, since the focus of our sponsored research was on aging-aircraft damage detection, ${ }^{23}$ our active-sensor experiments were conducted on thin metallic plates. However, the findings of this study can be easily extended to practical geometries and composite material structures. In this paper we explore a wide range of frequencies, from $10 \mathrm{kHz}$ into low $\mathrm{MHz}$, and discover an excitation "sweet spot" at $300 \mathrm{kHz}$ that is ideal for pulse-echo studies. The paper is organized in four main sections. In the first section, a general description of the piezoelectric active sensor and of the mechanism of its interaction with the host structure is presented. It is shown that, by applying a "pinching" action on the structural surface, the piezoelectric active sensor can excite elastic waves in the structural substrate. Conversely, the same sensor, which is intimately bonded to the structure, can also act as an elastic wave detector. This dual action (emitter and detector) imparts to it the "active-sensor" characteristics. In the second section, the theory of elastic wave propagation, with special focus on guided waves in thin-wall structures (Lamb waves), is briefly reviewed. The dispersive nature of the Lamb waves, and their symmetric and antisymmetric modes (pseudo-axial and pseudo-flexural waves, respectively) are presented and discussed. The third part of the paper is dedicated to theoretical and experimental beam studies. Finite-element simulations and experimental results on a long narrow strip constructed from thin-gage aircraftgrade aluminum stock are presented. Elastic wave generation and reception, and its reflection from boundaries, are used to study and experimentally verify the dispersion of the wave speed with the frequency-thickness product. Aspects of determining the optimal excitation frequency are discussed. The detection of structural damage (simulated cracks) is illustrated using the pulse-echo method. The fourth part of the paper is dedicated to plate studies. An array of 11 PWASs was placed in a grid pattern on a rectangular thin-gage aircraftgrade aluminum plate. Wave speed dispersion characteristics are verified, and the pulse-echo method is illustrated.

This study demonstrates that transmission and reception of guided elastic waves in thin-wall structures can be effectively implemented using surface-mounted small-size piezoelectric wafers acting as active-sensor devices. These guided waves can be used to scan the bi-dimensional topology of the thin walled structure like a "structural radar" and are able to detect internal damage through ultrasonic and acousto-ultrasonic techniques. The piezoelectric active sensors are inexpensive and unobtrusive. They can be deployed over large structural areas to create active sensor arrays that allow the application of phased array principles for beam steering and multidirectional detection.

\section{Piezoelectric Wafer Active Sensors}

PWASs are small, non-intrusive, and inexpensive piezoelectric wafers that are intimately affixed to the structure and can actively interrogate the structure. ${ }^{24}$ Commonly, they are manufactured from thin wafers of the piezoceramic $\mathrm{Pb}(\mathrm{Zr}$ $\mathrm{Ti}_{3} \mathrm{O}_{3}$ (a.k.a. PZT). Unlike conventional ultrasonic transducer, PWASs are non-resonant devices with wide-band capabilities. They can be wired into sensor arrays that are connected to data concentrators and wireless communicators. Piezoelectric active sensors have captured the interest of academic and industrial communities due to their low cost and small non-intrusive nature. ${ }^{25,26}$

The general constitutive equations of linear piezoelectric material behavior, given by ANSI/IEEE Standard 176$1987,{ }^{27}$ describe a tensorial relation between mechanical and electrical variables (mechanical strain $S_{i j}$, mechanical stress $T_{k l}$, electrical field $E_{k}$, and electrical displacement $D_{j}$ ) in the form

$$
\begin{aligned}
& S_{i j}=s_{i j k l}^{E} T_{k l}+d_{k i j} E_{k} \\
& D_{j}=d_{j k l} T_{k l}+\varepsilon_{j k}^{T} E_{k},
\end{aligned}
$$

where $s_{i j k l}^{E}$ is the mechanical compliance of the material measured at zero electric field $(E=0), \varepsilon_{j k}^{T}$ is the dielectric permittivity measured at zero mechanical stress $(T=0)$, and $d_{k i j}$ is the piezoelectric coupling between the electrical and mechanical variables. The direct piezoelectric effect is reflected in the second equation, while the first equation refers to the converse piezoelectric effect. In our study, we will consider a PWAS polarized across the thickness. In this configuration, mechanical stress and strain are applied in the 1- and 2-directions, i.e., in the plane of the surface, while the electric field acts in the 3-direction, i.e., normal to the surface. Hence, the significant electromechanical couplings for this type of analysis are the 31 and 32 effects. The application of an electric field, $E_{3}$, induces surface strains, $S_{11}$ and $S_{22}$, and vice versa. Figure 1(a) shows an active sensor consisting of a lead zirconate titanate (PZT) piezoceramic wafer affixed to the structural surface. The PWAS is directly connected to the source of electrical excitation through the connecting wires. The piezoelectric wafer is also intimately bonded to the structure, such that the strain/displacement compatibility and stress/force equilibrium principles apply. As the PZT material is electrically activated, strain is induced in the piezoelectric wafer, and interaction forces and moments appear at the interface between the sensor and the structure. In the pinforce model, the interaction force, $F_{P Z T}$, is assumed to act at the sensor boundary only. Induced by $F_{P Z T}$ are activation forces and moments $\left(N_{a}\right.$ and $\left.M_{a}\right)$, which apply a pinching action to the structural surface and generate structural waves (Fig. 1(b)):

$$
F_{P Z T}=\hat{F}_{P Z T} \mathrm{e}^{i \omega t}, N_{a}=F_{P Z T}, M_{a}=F_{P Z T} \frac{h}{2} .
$$

Conversely, when an elastic wave travels through the structure, the sensor becomes activated through the strain/displacement compatibility condition. In accordance with eq (1), the strain induced in the sensor generates an electric field that is captured as voltage at the sensor terminals. In the pin-force model, the sensor strain is proportional with the difference in displacement between its extremes. This 


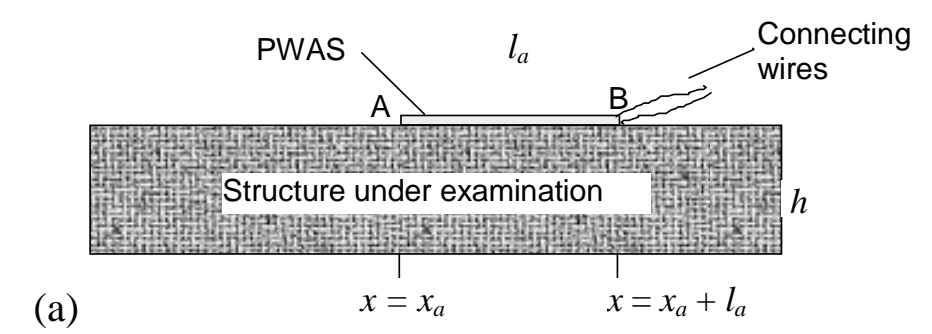

PZT-wafer strain and displacement, $\varepsilon_{\mathrm{PZT}}$ and $u_{\mathrm{PZT}}$

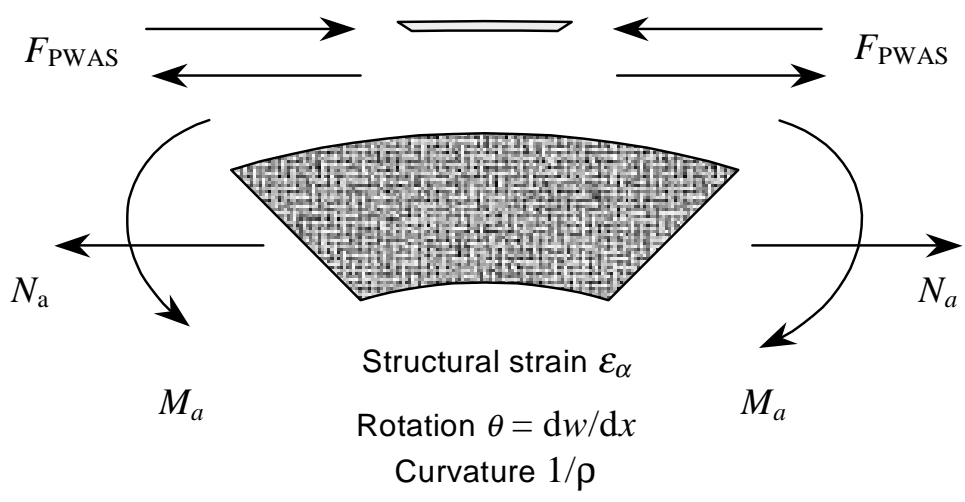

(b)

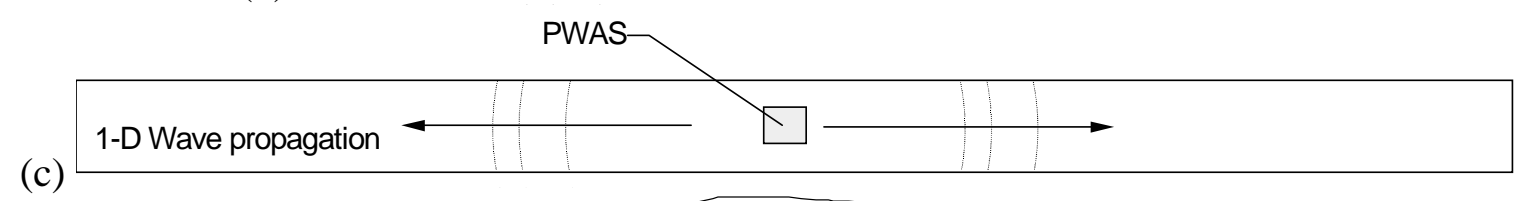

(d)

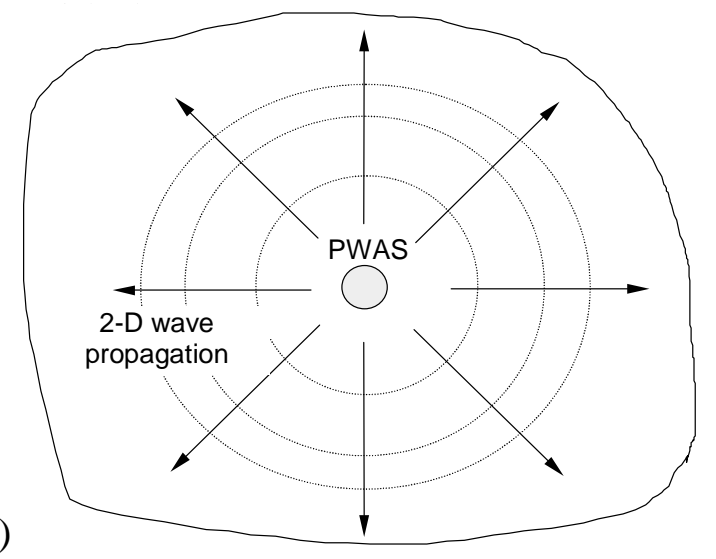

Fig. 1-PWAS interaction with host structure: (a) piezoelectric wafer transducer affixed to the host structure, (b) interaction forces and moments, (c) PWAS on a 1D structure, and (d) PWAS on a 2D structure

observation underpins the concept of "sensor tuning", i.e., optimal coupling between sensor and structure is achieved when the sensor effective length equals the half wavelength of the elastic wave in the structure $(a=\lambda / 2)$.

For a PWAS affixed to a one-dimensional (1D) structure, e.g., a beam (Fig. 1(c)), the wave propagation is mainly 1D. In this case, the dominant electromechanical coupling constant is $d_{31}$. If the active sensor is placed on a two-dimensional (2D) structure, the wave propagation is, in principle, 2D (Fig. 1(d)). Since the electromechanical coupling constants, $d_{31}$ and $d_{32}$, have essentially same value, radial symmetry can be applied, and the analysis can be reduced to a $1 \mathrm{D}$ case in the radial coordinate, $r .24$

\section{Review of Elastic Wave Propagation in Thin Plates}

The study of wave propagation in an elastic medium has a long history. Elastic waves in solid media can travel in a variety of modes, each associated with a wave type: axial, shear, classical flexure, Rayleigh, Lamb, Love, etc. ${ }^{28-30}$ Studies of the particle displacement in each mode type have been performed to understand the coupling between particle motion and the PWAS. Particle displacement animations are available at our website http://www.me.sc.edu/research/lamss under research Thrust 1. Elastic waves propagating in thin plates with free boundaries are called Lamb waves. Lamb waves are also known as guided plate waves, since the wave energy 


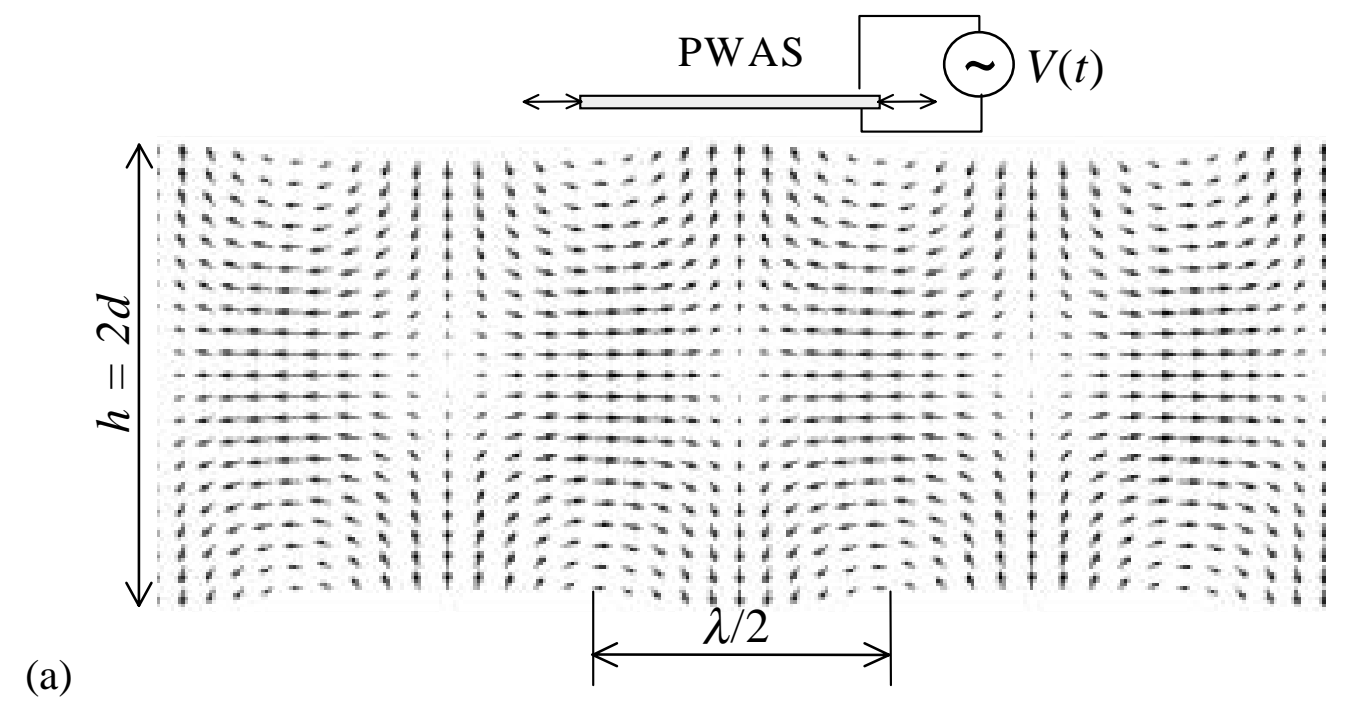

(a)
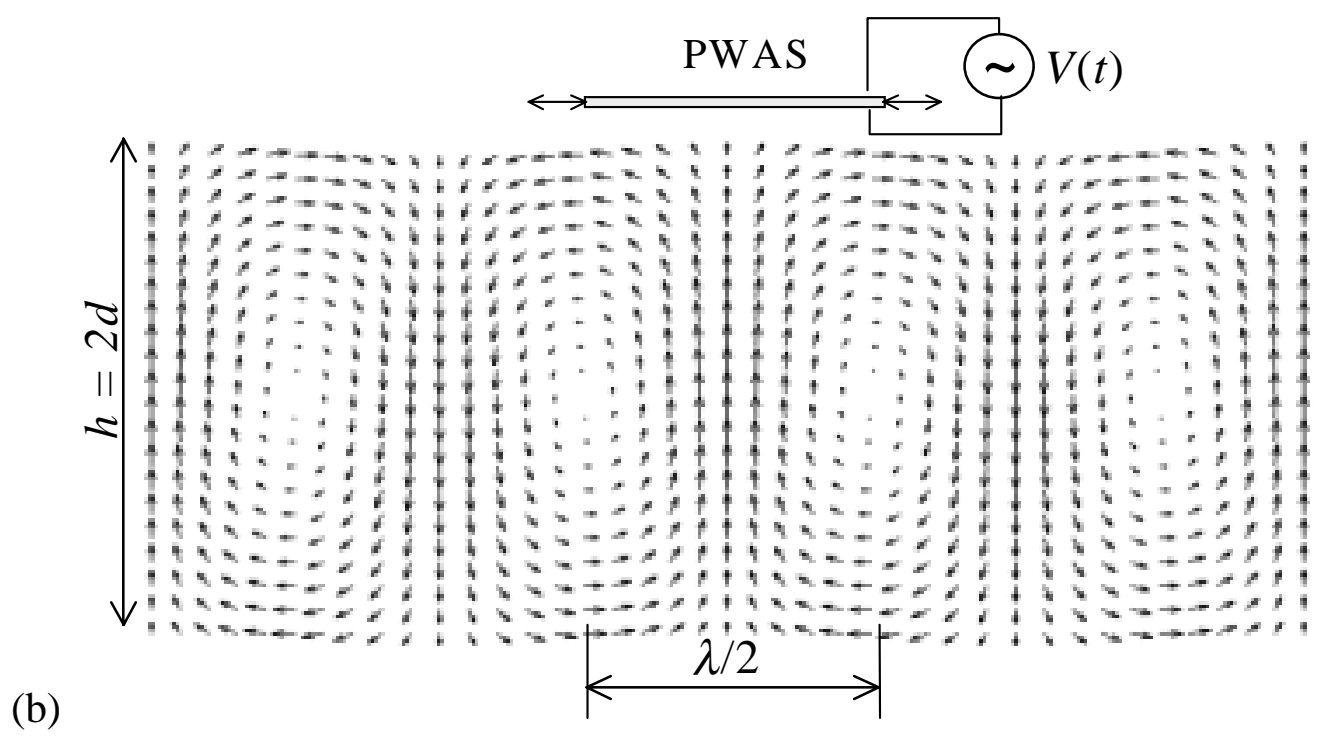

Fig. 2-Simulation of PWAS interactions with Lamb modes: (a) symmetric Lamb mode $\mathrm{S}_{0}$, and (b) antisymmetric Lamb mode $A_{0}$. For full animation, see http://www.me.sc.edu/research/lamss/research/Waves/sld004.htm

remains contained within the thin-plate guide. The Lamb wave particle displacements are both along the plate and across the thickness. Lamb waves can propagate in a number of modes, either symmetrical or antisymmetrical with respect to the thickness mid-surface (Fig. 2).

\section{Symmetric Lamb Waves}

To find the eigenvalues of the symmetrical Lamb waves, we solve the following form of the Rayleigh-Lamb frequency equation $^{29}$

$$
\frac{\tan \left(\sqrt{1-\zeta^{2}} \bar{d}\right)}{\tan \sqrt{\xi^{2}-\zeta^{2}}}+\frac{4 \zeta^{2} \sqrt{1-\zeta^{2}} \sqrt{\xi^{2}-\zeta^{2}}}{\left(2 \zeta^{2}-1\right)^{2}}=0
$$

where $\xi=\sqrt{c_{S}^{2} / c_{P}^{2}}, \zeta=\sqrt{c_{S}^{2} / c_{L}^{2}}, c_{L}$ is the Lamb wave speed, $\bar{d}=k_{S} d, d$ is the half thickness of the plate, and $k_{S}$ $=\omega / c_{S}$. By solving the Rayleigh-Lamb eq (3), we can determine the Lamb wave speed, $c_{L}$. According to eq (3), the Lamb wave speed depends on the non-dimensional thickness, $\bar{d}$, i.e., on the thickness-frequency product. Equation (3) admits several roots, corresponding to several symmetrical Lamb wave modes, called $S_{0}, S_{1}$, etc. A plot of the $S_{0}$ Lamb wave speed versus frequency is given in Fig. 3(a). The calculation was made for an aluminum plate, with a thickness value $2 d=1.6 \mathrm{~mm}$. The roots of eq (3) were found numerically using mathematical software. Examination of Fig. 3(a) indicates that, at low frequencies $(f<500 \mathrm{kHz})$ the speed of the symmetric Lamb wave approaches the speed of axial 


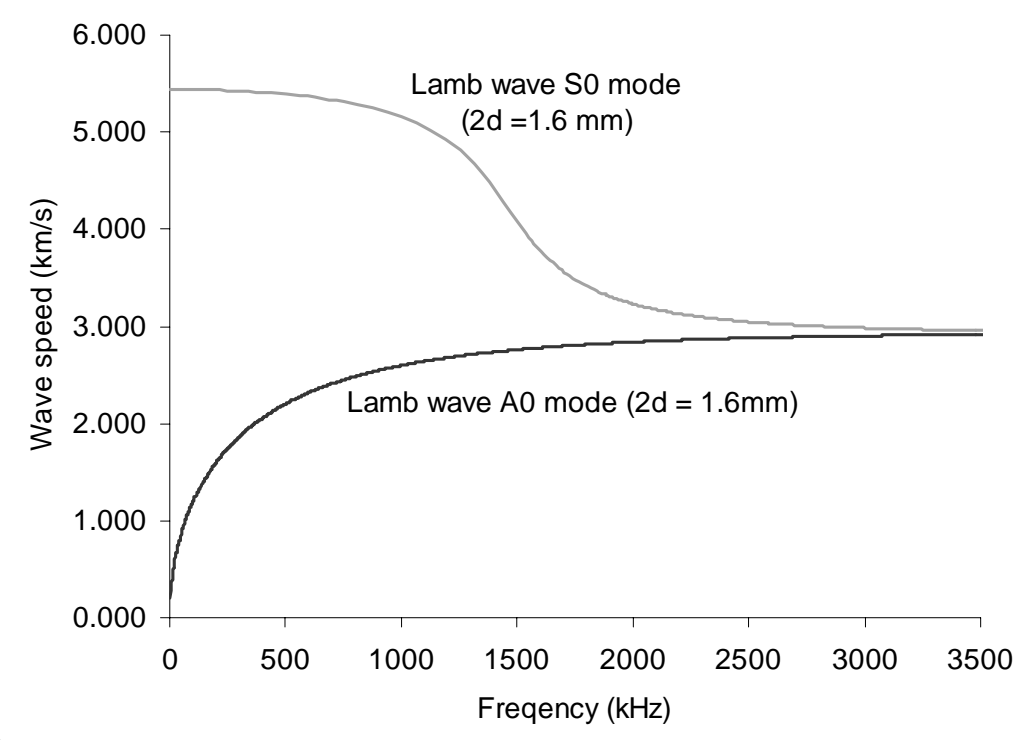

(a)

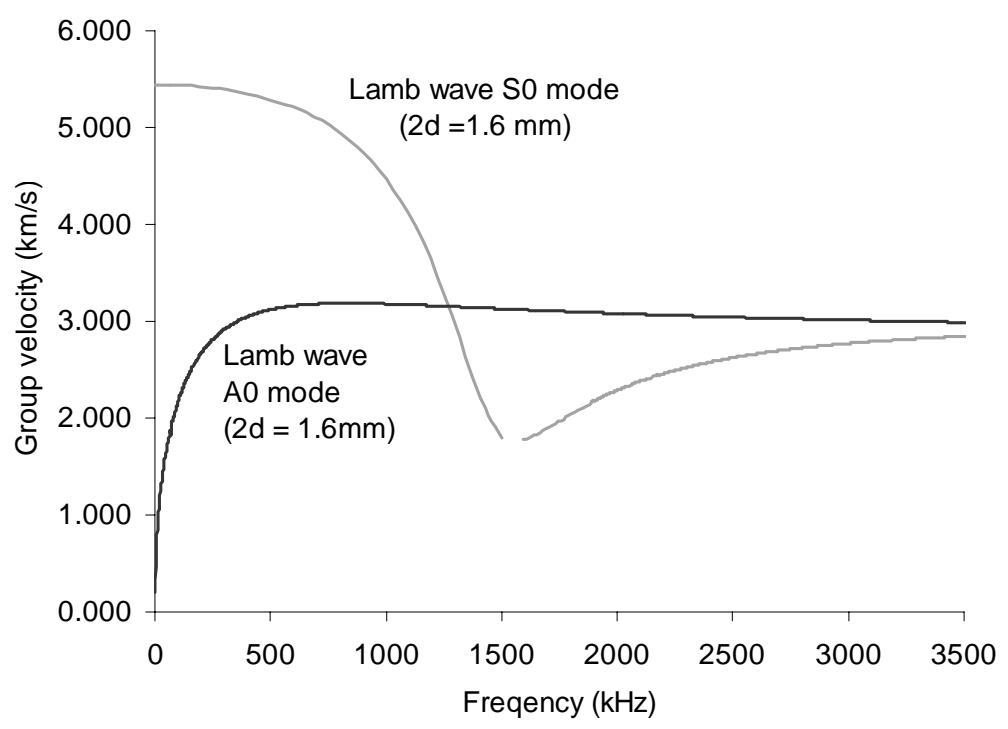

(b)

Fig. 3-Dispersion curves for Lamb waves in $1.6 \mathrm{~mm}$ aluminum alloy plates: (a) wave speed, and (b) group velocity

waves, $c_{P}$. The particle motion for a symmetric Lamb wave is given by ${ }^{29}$

$$
\begin{aligned}
u(x, z, t)= & R e\left[A k _ { L } \left(\frac{\cosh (q z)}{\sinh (q d)}\right.\right. \\
& \left.\left.-\frac{2 q s}{k_{L}^{2}+s^{2}} \frac{\cosh (s z)}{\sinh (s d)}\right) e^{i\left(k_{L} x-\omega t \frac{\pi}{2}\right)}\right] \\
w(x, z, t)= & \operatorname{Re}\left[A q \left(\frac{\sinh (q z)}{\sinh (q d)}\right.\right. \\
& \left.\left.-\frac{2 k_{L}^{2}}{k_{L}^{2}+s^{2}} \frac{\sinh (s z)}{\sinh (s d)}\right) e^{i\left(k_{L} x-\omega t\right)}\right]
\end{aligned}
$$

where $k_{L}=\omega c_{L}$, and $q=\sqrt{k_{L}^{2}-k_{P}^{2}}, s=\sqrt{k_{L}^{2}-k_{S}^{2}}$. A plot of the particle motion defined by eqs (4) and (5) for the $\mathrm{S}_{0}$ mode is given in Fig. 2(a) for the $\mathrm{S}_{0}$ mode. The symmetry of the motion with respect to the mid-surface is apparent.

\section{Antisymmetric Lamb Waves}

For antisymmetric Lamb waves, the Rayleigh-Lamb frequency equation takes the form

$$
\frac{\tan \left(\sqrt{1-\zeta^{2}} \bar{d}\right)}{\tan \sqrt{\xi^{2}-\zeta^{2}}}+\frac{\left(2 \zeta^{2}-1\right)^{2}}{4 \zeta^{2} \sqrt{1-\zeta^{2}} \sqrt{\xi^{2}-\zeta^{2}}}=0 .
$$

Equation (6) admits several roots, corresponding to several antisymmetrical Lamb wave modes, called $\mathrm{A}_{0}, \mathrm{~A}_{1}$, etc. The plot of the Lamb wave speed branch $\mathrm{A}_{0}$ is also presented in Fig. 3(a). At high frequencies $(2500 \mathrm{kHz}<f)$, the dispersion curves for the $\mathrm{S}_{0}$ and $\mathrm{A}_{0}$ modes coalesce. The particle motion 


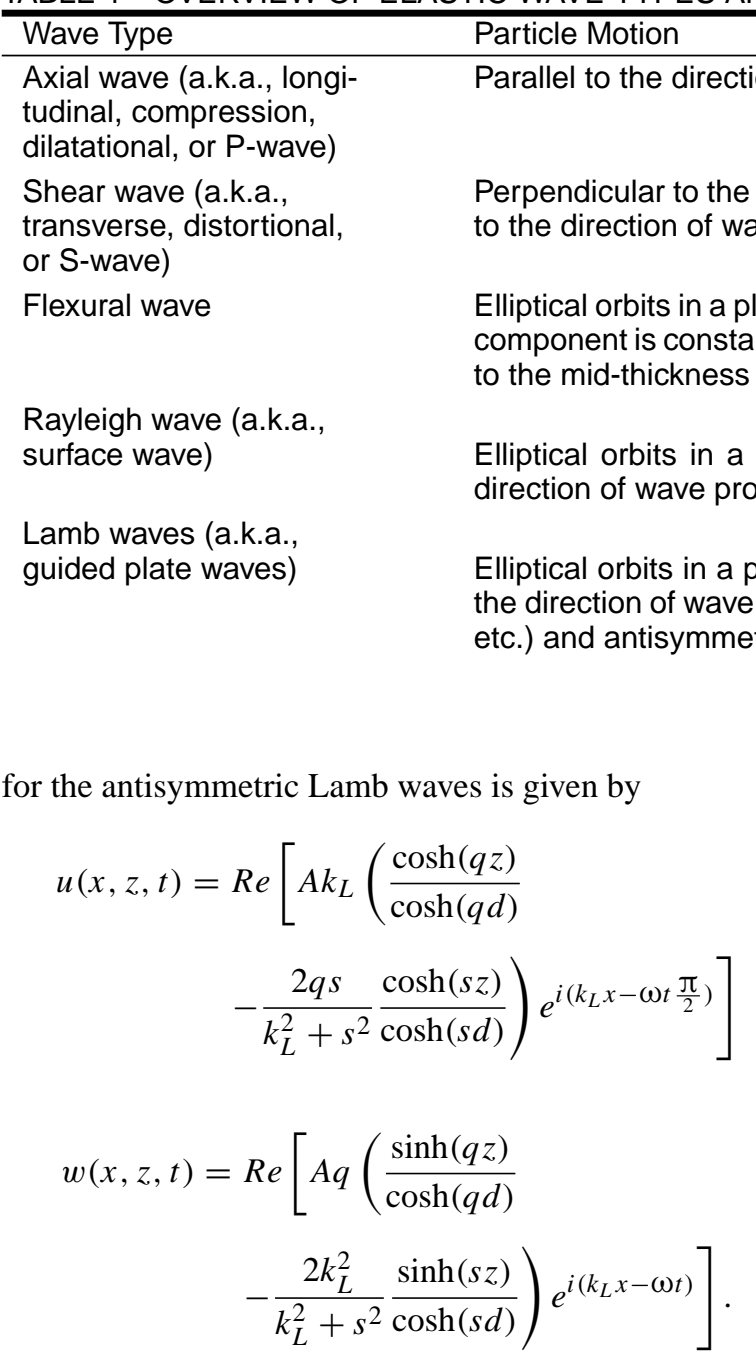

A plot of the particle motion defined by eqs (7) and (8) for the $\mathrm{A}_{0}$ mode is given in Fig. 2(b). The antisymmetry of the motion with respect to the mid-surface is apparent.

\section{Group VELocity of LAMB WAVES}

The group velocity of Lamb waves is important when examining the traveling of Lamb wave packs, as in the experiments described later in this paper. To calculate the group velocity of Lamb waves, we used the formula ${ }^{4}$

$$
c_{g}=c^{2}\left[c-(f d) \frac{\mathrm{d} c}{\mathrm{~d}(f d)}\right]^{-1}
$$

where $c$ is the Lamb wave phase velocity. Figure 3(b) shows a plot of the group velocities versus frequency for thin-gage aluminum with thickness $2 d=1.6 \mathrm{~mm}$. Shown in Fig. 3(b) are the group velocities for symmetric Lamb waves $\left(\mathrm{S}_{0}\right)$, and antisymmetric Lamb waves $\left(\mathrm{A}_{0}\right)$. Table 1 gives a comparative overview of the common wave types and their particle motion.

\section{Embedded Ultrasonics on 1D Beam Structures}

In the beginning, our investigation was focused on simple 1D wave-propagation studies that allow a simpler analysis and foster rapid understanding. For our studies, we selected a long and narrow thin-gage metallic strip that resembles a long beam. The beam was instrumented with five pairs of piezoelectric active sensors and modeled with the finite-element method (FEM). Details of our investigation are presented next.

\section{D Specimen Description}

The 1D beam specimen considered in our study was made from $1.6 \mathrm{~mm}$ thick aircraft-grade 2024 aluminum alloy. The beam was $914 \mathrm{~mm}$ long, $14 \mathrm{~mm}$ wide, and $1.6 \mathrm{~mm}$ thick. PWASs ( $7 \mathrm{~mm}$ square, $0.2 \mathrm{~mm}$ thick) were installed in pairs (i.e., on both sides of the beam thickness) at five locations (A-E), as shown in Fig. 4 and Table 2. The double-sided installation was chosen to permit symmetric and antisymmetric excitation. Thus, axial and flexural waves could be selectively generated.

\section{Excitation Signal}

The excitation signal considered in our studies consisted of a smoothed tone burst. The smoothed tone burst was obtained from a pure tone burst of frequency $f$ filtered through a Hanning window. The Hanning window was described by the equation:

$$
x(t)=\frac{1}{2}\left[1-\cos \left(2 \pi t / T_{H}\right)\right], \quad t \in\left[0, T_{H}\right] .
$$

The number of counts, $N_{B}$, in the tone bursts matches the length of the Hanning window, i.e.,

$$
T_{H}=N_{B} / f \text {. }
$$

The tone burst excitation was chosen in order to excite coherent single-frequency waves. This aspect is very important especially when dealing with dispersive waves (flexure,

TABLE 2-LOCATIONS OF SENSORS ON THE THIN NARROW-STRIP BEAM SPECIMEN

\begin{tabular}{cccccc}
\hline & \multicolumn{5}{c}{ Sensor } \\
\cline { 2 - 6 } & $\mathrm{A}$ & $\mathrm{B}$ & $\mathrm{C}$ & $\mathrm{D}$ & $\mathrm{E}$ \\
\hline$x(\mathrm{~mm})$ & 57 & 257 & 457 & 657 & 857 \\
$y(\mathrm{~mm})$ & 7 & 7 & 7 & 7 & 7
\end{tabular}


HP 33120

Signal generator

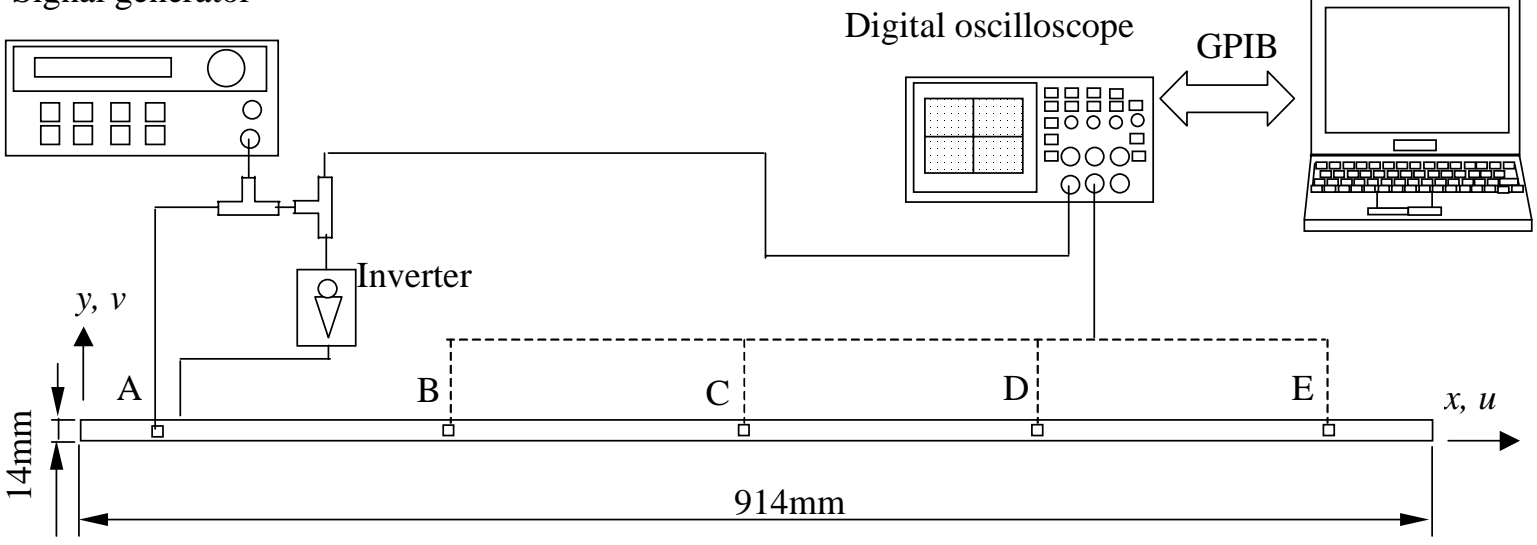

Fig. 4-1D beam specimen consisting of a narrow strip of $1.6 \mathrm{~mm}$ thick 2024 aluminum alloy, $14 \mathrm{~mm}$ wide and $914 \mathrm{~mm}$ long. Five pairs of PWASs (A-E) are also shown.

Rayleigh, Lamb, Love, etc.). The Hanning window smoothing was applied to reduce the excitation of frequency side lobes associated with the sharp transition at the start and the end of a conventional tone burst. Through these means, it was intended that the dispersion effects would be minimized and the characteristics of elastic wave propagation would be readily understood. Figure 5 shows a comparison of raw and smoothed $10 \mathrm{kHz}$ tone bursts, as well as their Fourier transform. It is apparent that, although both tone bursts excite the same central frequency of $10 \mathrm{kHz}$, the raw tone burst (Fig. 5(a)) also excites a considerable number of side lobes, below and above the central frequency. By contrast, the smoothed tone burst (Fig. 5(b)) does not produce side lobes.

The smoothed tone burst that resulted from this process was numerically synthesized and stored in PC memory as the excitation signal. This numerically generated excitation signal was used in the finite-element analysis and in the experimental investigation.

\section{Finite Element Analysis of Wave Propagation in a Beam Specimen}

Numerical simulations of the wave propagation process were performed using the commercially available finiteelement code, ANSYS. The narrow-strip beam specimen was modeled using four-node shell elements (SHELL63). The element has six degrees of freedom at each node. The shell element displayed both bending and membrane capabilities. The discretization process used 3976 elements, arranged in rows of four across the beam width (Fig. 6). The FEM also permitted the simulation of structural defects, e.g., throughthickness cracks of length $2 a$. Such cracks were simulated through the nodal release method. Figure 6 shows a simulated crack placed transverse to the beam, along the transverse symmetry line. The crack length is dictated by the number of released nodes. In order to obtain better resolution for crack simulation, the discretization mesh was locally refined, as indicated in Figure 6.

Two forms of the elastic wave propagation were studied: the flexural waves and the axial waves. To attain wave exci- tation, we considered creating situations in the finite-element model that are equivalent to the excitation applied by the piezoelectric wafer. First, we identified groups of finite elements that cover areas geometrically equivalent to that covered by the PWAS installed on the specimen (A-E in Fig. 4). Thus, we designated areas in the finite-element model that represent active sensors. Then, to generate waves, we applied prescribed displacements to the nodes delimiting the contour of these active areas. Consistent with the physical phenomenon, the displacement applied to nodes representing opposite ends of the piezoelectric wafer had to be in opposite phase. This ensures that the net effect on the structure is self-equilibrating. To generate axial waves, we applied nodal translations. While for flexural waves, we applied nodal rotations. The detection of the elastic waves followed the same general principle as that applied to wave generation. The variables of interest were the differences between the displacements at the opposing ends of the active sensor, i.e., $\Delta u$ for axial waves, and $\Delta w^{\prime}$ for flexural waves.

\section{SIMULATION OF AXIAL WAVES}

Figure 7 shows FEM simulation of axial waves in the beam excited with a $100 \mathrm{kHz}$ five-count Hanning-windowed axial burst at the left-hand end of the beam. The patterns of dilatation and contraction (in-plane motion) are shown. The wave was captured after traveling for $50 \mu$ s. Figure 7(a) gives an overall view, while Fig. 7(b) gives a magnified detail of the first quarter of the beam. We note that the number of peaks in the wave is greater than the burst count of five because both the incident wave and the wave reflected from the left-hand end of the beam are superposed in this wave front. Figure 8 shows the wave signal received at sensors A-E. It is easily appreciated how the wave travels down the beam from $\mathrm{A}$ to $\mathrm{E}$, then reflects at the right-hand end, and returns to $\mathrm{A}$, followed by repetition of this pattern. We observe that, at the beginning, the five counts of the smoothed tone-burst excitation can be readily identified. As the wave travels further and undergoes reflections, its coherence somehow diminishes, and some dispersion occurs. This loss of coherence may also be attributed to accumulation of numerical error. 

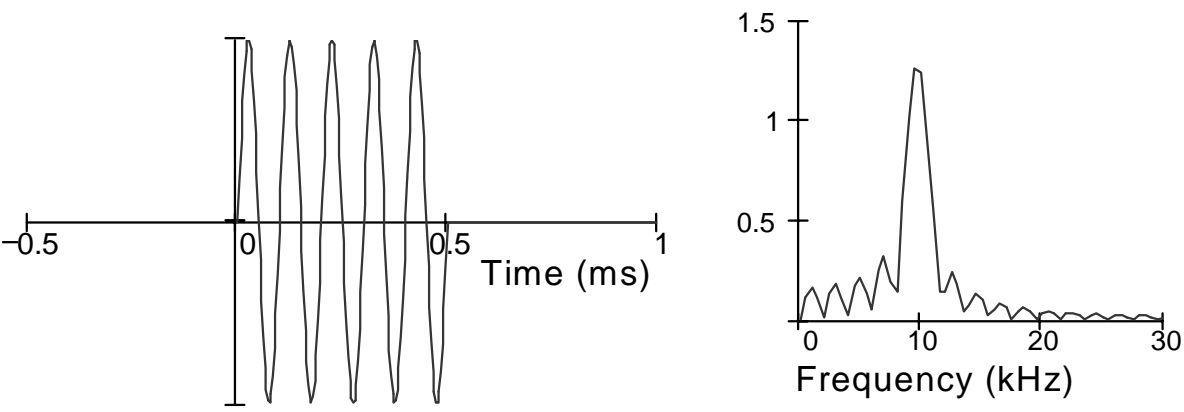

(a)

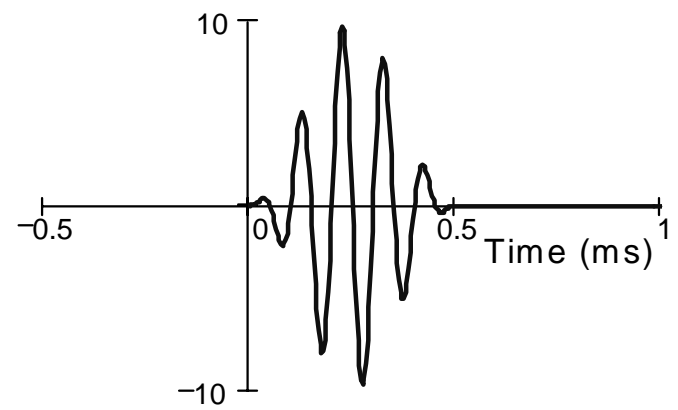

(b)

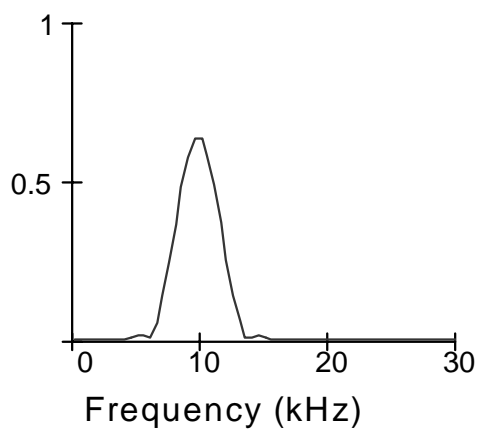

Fig. 5-Example of $10 \mathrm{kHz}$, five-count tone-burst excitation: (a) raw tone burst, and (b) smoothed tone burst

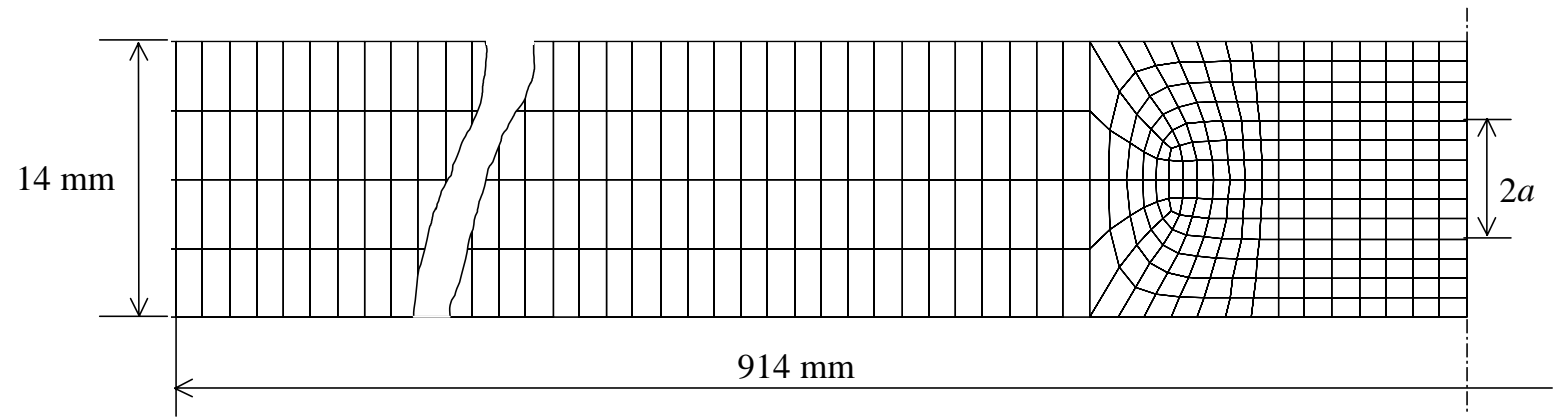

Fig. 6-The mesh used in the finite-element modeling was refined around the region with a simulated crack. The left-hand side half of the symmetric beam is shown. The crack is simulated along the symmetry line of the beam, i.e., at the right-hand end of the drawing. The crack length, $2 a$, is dictated by the number of released nodes.

(a)

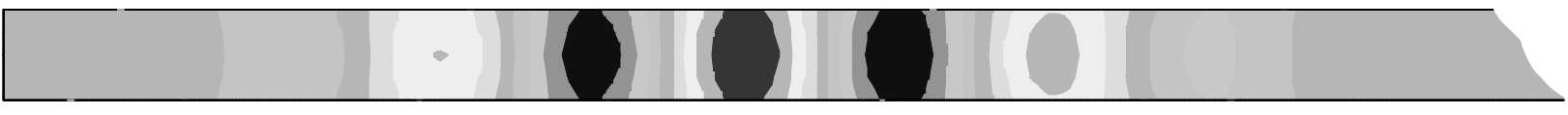

(b)

Fig. 7-FEM simulation of axial waves in the $914 \times 14 \times 1.6 \mathrm{~mm}^{3}$ aluminum alloy beam excited with $100 \mathrm{kHz}$ five-count Hanning-windowed axial burst at the left-hand end. The wave is captured after traveling for $50 \mu \mathrm{s}$ : (a) overall view, and (b) details of first quarter of the beam. The number of peaks in the wave is greater than five because both the incident wave and the wave reflected from the left-hand side end of the beam are superposed in this wave front. 


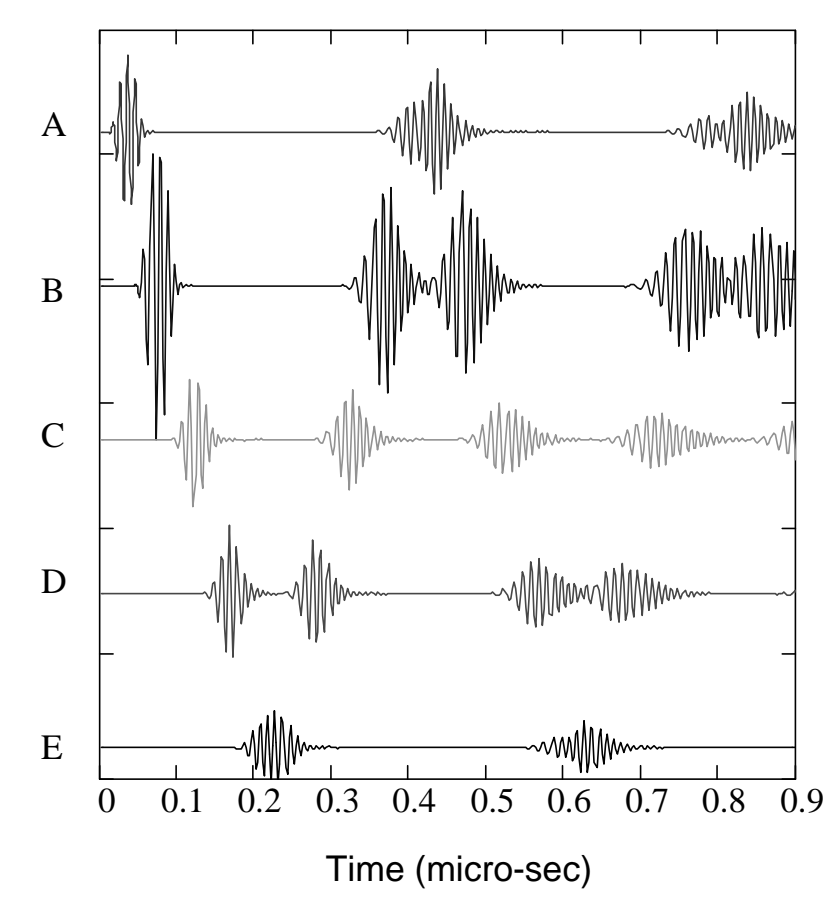

Fig. 8-FEM simulation of the axial wave signals received at sensors A-E (100 kHz five-count smoothed tone-burst excitation at active sensor $A$ ).

Figure 9 shows the FEM simulation of the pulse-echo method used for damage detection. The active sensor placed at the left-hand end was used to send a $100 \mathrm{kHz}$ five-count Hanning-windowed axial burst and to receive the elastic wave responses. In a beam without crack (Fig. 9(a)), the initial signal and the reflection from the right-hand end appear. If an $8 \mathrm{~mm}$ through-the-thickness transverse crack is simulated in the center of the beam, the reflection (echo) from this crack also appears (Fig. 9(b)). As the crack length increases, the amplitude of the reflection increases (Fig. 9(c)).

\section{SiMULATION OF FLEXURAL WAVES}

Figure 10 shows the FEM simulation of flexural waves in the beam excited with a $100 \mathrm{kHz}$ five-count Hanningwindowed axial burst at the left-hand end. The peaks and valleys of flexural wave propagation are apparent. The wave was captured after traveling for $99.3 \mu \mathrm{s}$. Figure 10(a) gives an overall view, while Fig. 10(b) gives magnified detail of the first quarter of the beam. We note that the number of peaks in the wave is greater than the burst count of five because both the incident wave and the wave reflected from the left-hand end of the beam are superposed in this wave front. Figure 11 shows the wave signal received at sensors A-E for a $10 \mathrm{kHz}$ excitation. It is easily appreciated how the wave travels down the beam from $\mathrm{A}$ to $\mathrm{E}$, then reflects at the right-hand end, and returns to A, followed by repetition of this pattern. We observe that the five counts of the smoothed tone-burst excitation can be readily identified in the initial signal, but this pattern quickly deteriorates due to wave dispersion. As the wave travels further and undergoes reflections, its coherence further diminishes. The loss of coherence may also be attributed to accumulation of numerical error.
Figure 12 shows the FEM simulation of the pulse-echo method used for damage detection. The active sensor placed at the left-hand end was used to send a $100 \mathrm{kHz}$ five-count Hanning-windowed axial burst and to receive the elastic wave responses. In a beam without a crack (Fig. 12(a)), the initial signal and the reflection from the right-hand end appear. If an $8 \mathrm{~mm}$ through-the-thickness transverse crack is simulated in the center of the beam, the reflection from this crack also appears (Fig. 12(b)). As the crack length increase, the amplitude of the reflection increases (Fig. 12(c)).

\section{Comparison betWeEn Axial and FleXuRAL WaVE SIMULATION RESULTS}

The main differences between using the pulse-echo method with axial waves versus flexural waves are revealed by the comparison of Figs. 9 and 12. Figures 9(a) and 12(a) show that, in this thin-strip beam specimen, the flexural wave speed at $100 \mathrm{kHz}$ is roughly half of the axial wave speed, since the same distance of $914 \mathrm{~mm}$ is traveled in roughly twice the time. In addition, the flexural wave echo of Fig. 12(a) shows a dispersion pattern (i.e., superposition of several waves with different frequencies), while the axial wave echo of Fig. 9(a) shows a much more coherent pattern. This is in agreement with the dispersion curves of modes $\mathrm{A}_{0}$ and $\mathrm{S}_{0}$ shown in Fig. 7 , since, at this frequency, the flexural waves, $A_{0}$, are much more dispersive than the axial waves, $S_{0}$. Figures $9(b)$ and 12(b) show that, for partial cracks of the same crack size $(8 \mathrm{~mm})$, the axial wave echo is much stronger than the flexural wave echo. Figures 9(c) and 12(c), on the other hand, show that for a large crack $(14 \mathrm{~mm})$ the flexural wave echo has increased much more than the axial wave echo. These observations indicate that both the axial and the flexural waves can offer advantages, under appropriate circumstances, for damage detection, and that both should be retained for further studies.

\section{THE IMPORTANCE OF HIGH FREQUENCY EXCITATION}

Although the results shown above refer mainly to one frequency $(100 \mathrm{kHz})$, our wave propagation simulation efforts were performed for a variety of frequencies in the range 10 $100 \mathrm{kHz}$. It has been found that the lower frequency limit was easier to simulate since at low frequencies the wavelength is longer and spans more finite elements, and hence the distortion of each element is less severe. Consequently, at low frequency, we could use larger elements, i.e., a coarser mesh, and less computation time. However, low-frequency waves are inappropriate for ultrasonic applications. To achieve damage detection with the pulse-echo method, the timewise length of the wave pack must be much less than the time taken for the echo to return. We observed that, at low frequencies (e.g., $10 \mathrm{kHz}$ ), the echo signal starts to appear before the incident signal has finished developing, thus markedly impeding the damage detection process. Hence, high excitation frequencies are needed. As the frequency increases, the wavelength decreases, and hence a finer finite-element mesh is needed to capture the wave propagation process, which increases considerably the computational effort. This was especially apparent for flexural waves, which, at a given frequency, have a shorter wavelength than axial waves. After considerable studies, the compromised frequency of $100 \mathrm{kHz}$ was selected. With this frequency, we were able to successfully simulate both axial and flexural wave patterns, and to clearly identify 


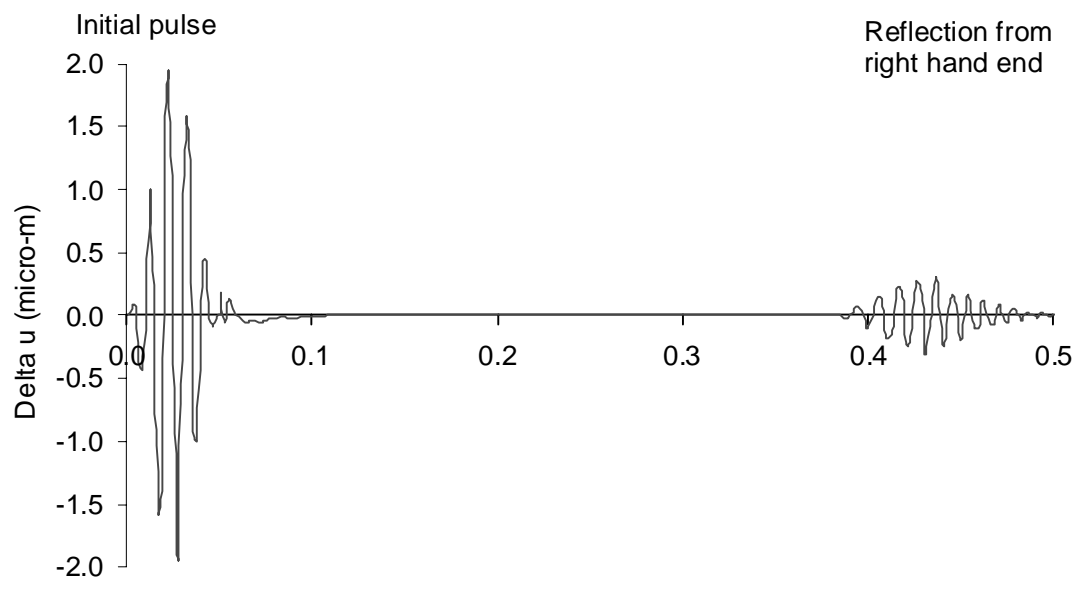

Time (ms)

(a)

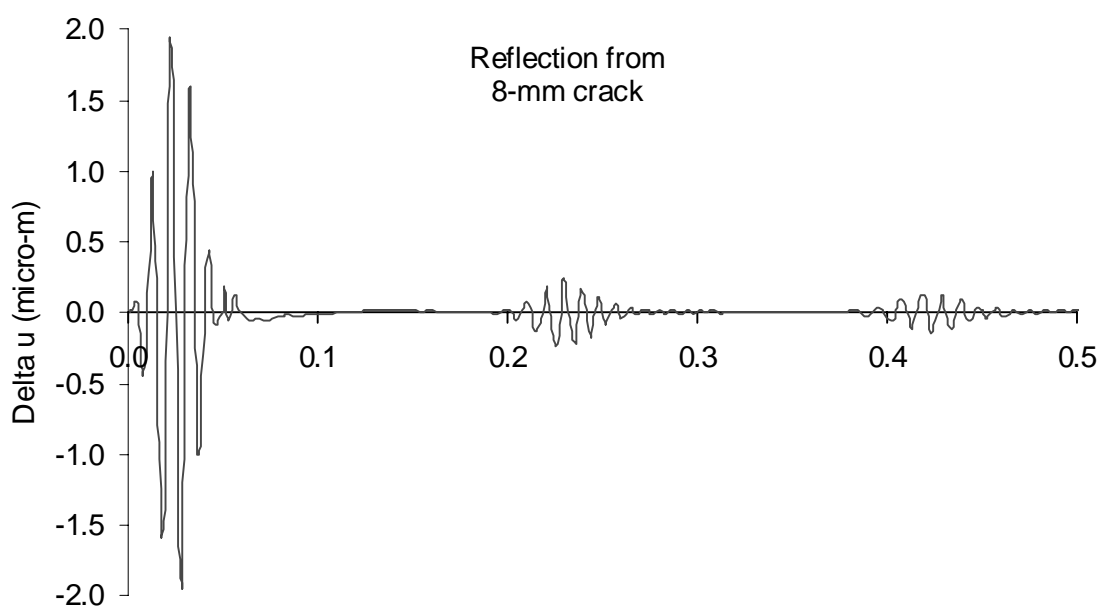

(b)

Time (ms)

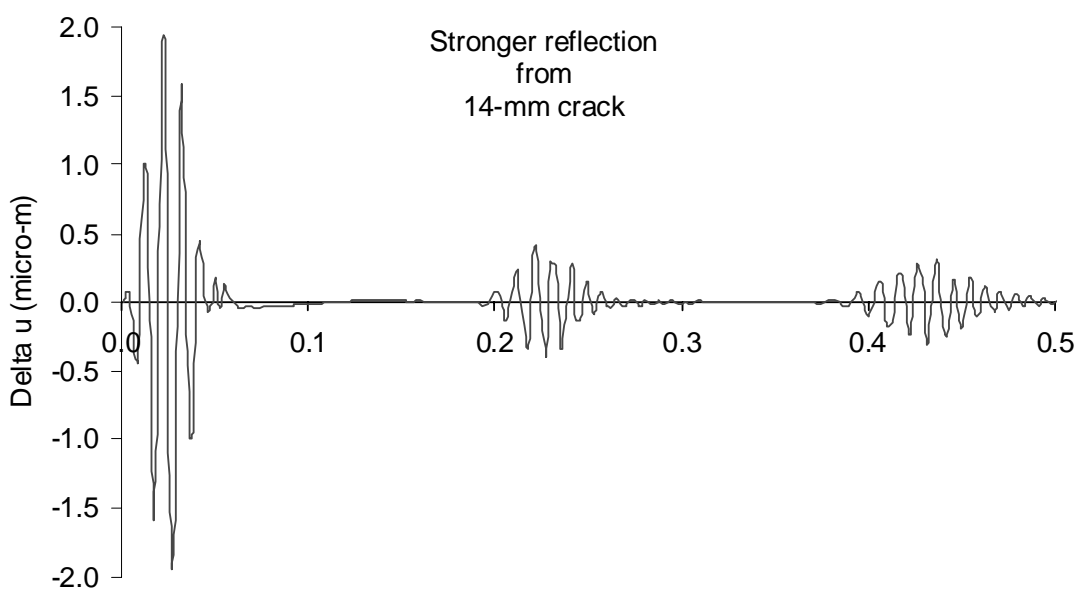

(c)

Fig. 9-FEM simulation of pulse-echo method in a $914 \times 14 \times 1.6 \mathrm{~mm}^{3}$ aluminum alloy beam using axial waves. A $100 \mathrm{kHz}$ fivecount Hanning-windowed axial burst was applied at the left-hand end. (a) The beam without a crack shows only the reflection from the right-hand end, (b) the beam with an $8 \mathrm{~mm}$ long through-the-thickness transverse crack shows, in addition, the reflection from the crack, (c) a longer crack $(14 \mathrm{~mm})$ gives a stronger reflection 


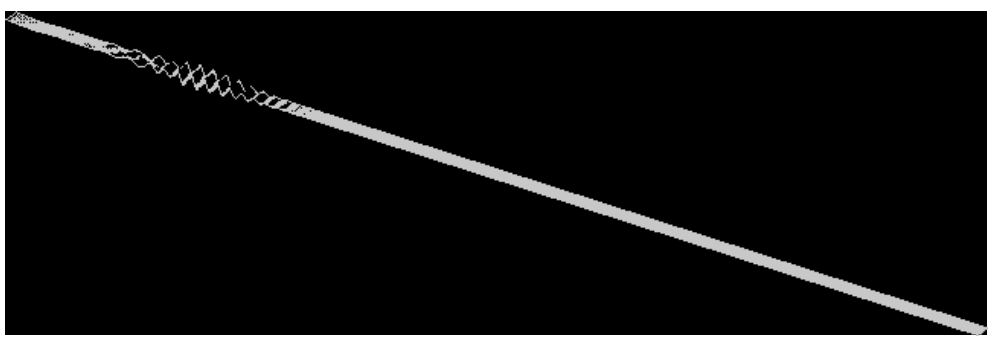

(a)

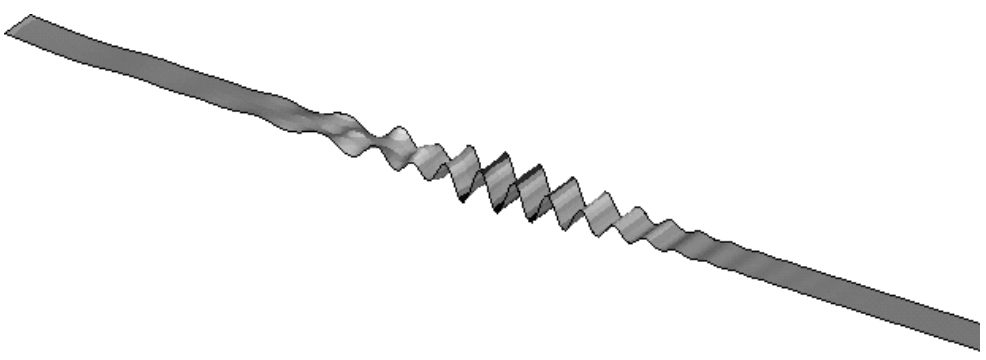

(b)

Fig. 10-FEM simulation of flexure waves in the $914 \times 14 \times 1.6 \mathrm{~mm}^{3}$ aluminum alloy beam excited with $100 \mathrm{kHz}$ five-count Hanning-windowed burst at the left-hand end. The wave is captured after traveling for $99.3 \mu s$ : (a) overall view, and (b) details of first quarter of the beam. The number of peaks in the wave is greater than five because both the incident wave and the wave reflected from the left-hand side end of the beam are superposed in this wave front

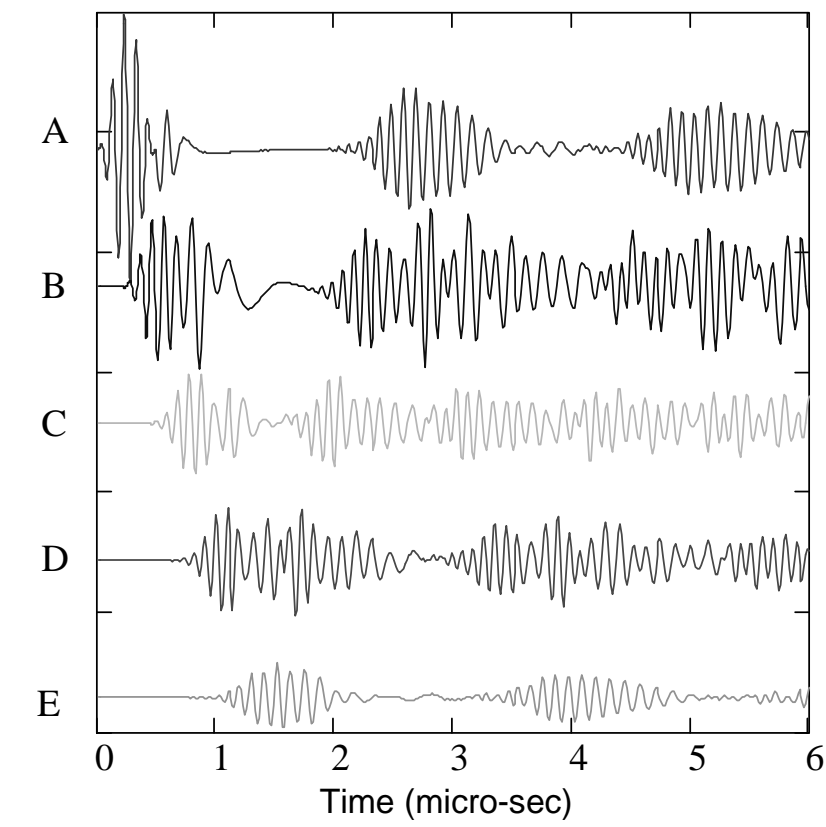

Fig. 11-FEM simulation of flexural wave signals received at sensors $A-E(10 \mathrm{kHz}$ five-count smoothed tone-burst excitation at active sensor $A$ )

defect-generated echoes as close as $100 \mathrm{~mm}$ from the source. For the detection of defects closer than $100 \mathrm{~mm}$, higher frequencies are required.

\section{Experimental Investigation of Wave Propagation in a Beam Specimen}

The experimental setup consisted of a HP 33120A signal generator, a Tektronix TDS 210 digital oscilloscope, and a laptop computer connected through a GPIB interface. The HP 33120A signal generator was used to generate constantamplitude tone bursts of three to five counts. The Tektronix TDS 210 digital oscilloscope connected to the laptop computer through the GPIB interface was used to collect data. The burst signal from the signal generator of $10 \mathrm{~V}$ peak-to-peak (pp) was applied directly to the PWAS. The signal was applied either to one of the two PWASs in the pair (one-sided excitation) or to both (two-sided excitation). In the latter case, both in-phase and out-of-phase excitation coupling between the upper and the lower PWAS was applied. To achieve out-ofphase coupling, the signal was split, and then routed through an operational amplifier inverter circuit. Through in-phase and out-of-phase excitation, the enhancement and suppression of certain wave types (axial, flexural) could be manipulated, and thus their properties could be separately studied. One of the five pairs of PWASs (A-E) was used for transmission, while the others were used for reception. Various combinations of transmission-reception locations were explored in a round-robin fashion.

Figure 13 shows several signal samples captured during this investigation. On each plot, signal A represents the excitation signal applied to the PWAS at location A, while B-E represent the reception signals picked up by the PWAS at locations B-E. The excitation signal was a $10 \mathrm{kHz}$, three-count constant-amplitude tone burst of $10 \mathrm{Vpp}$. The reception signals were in the $100 \mathrm{mVpp}$ range. Figure 13(a) presents the results of the excitation being applied to the upper PWAS at 
(a)

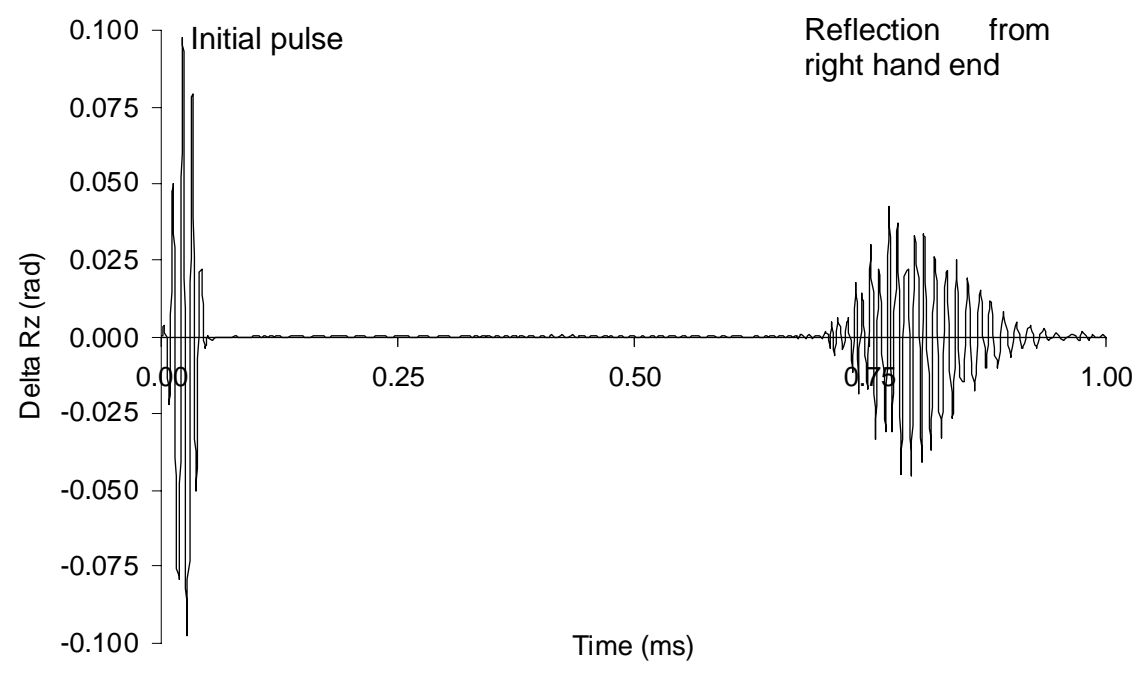

(b)

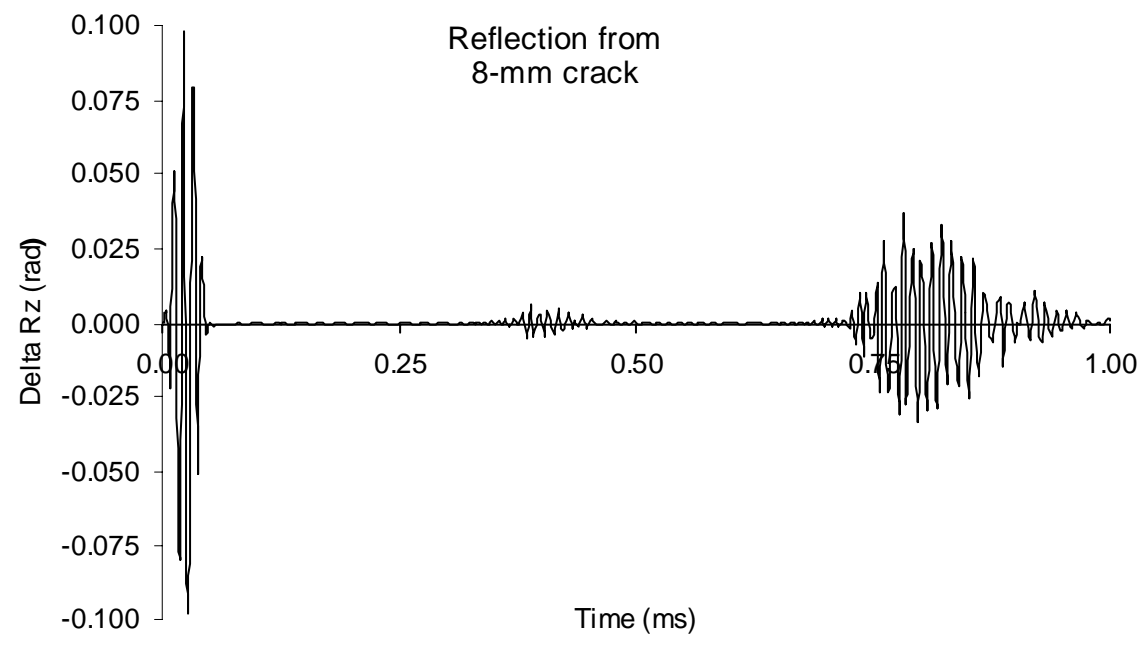

(c)

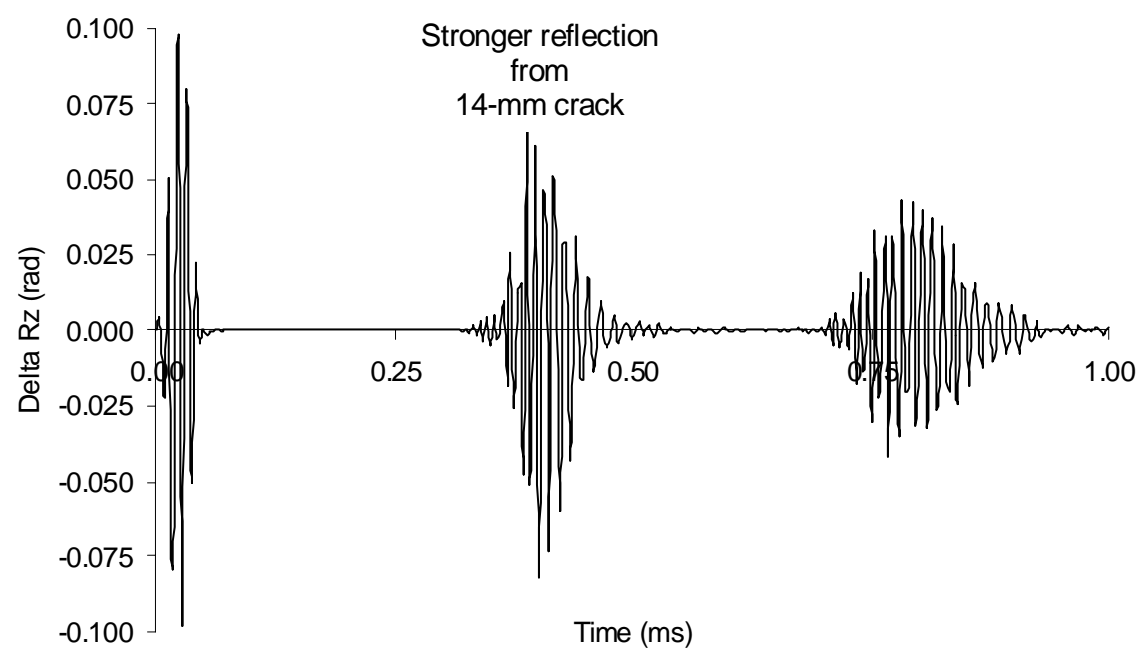

Fig. 12-FEM simulation of pulse-echo method in a $914 \times 14 \times 1.6 \mathrm{~mm}^{3}$ aluminum alloy beam using flexural waves. A 100 $\mathrm{kHz}$ five-count Hanning-windowed flexural burst was applied at the left-hand end. (a) The beam without a crack shows only the reflection from the right-hand end, (b) the beam with an $8 \mathrm{~mm}$ long through-the-thickness transverse crack shows, in addition, the reflection from crack, and (c) a longer crack $(14 \mathrm{~mm})$ gives a stronger reflection 


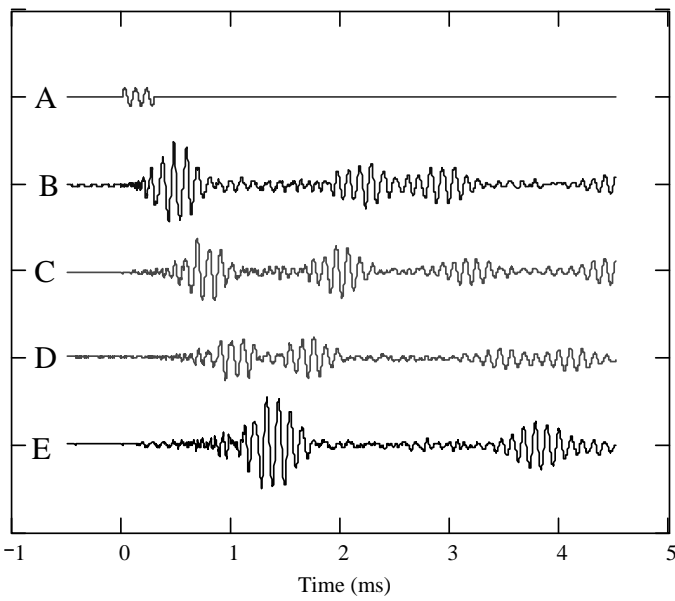

(a)

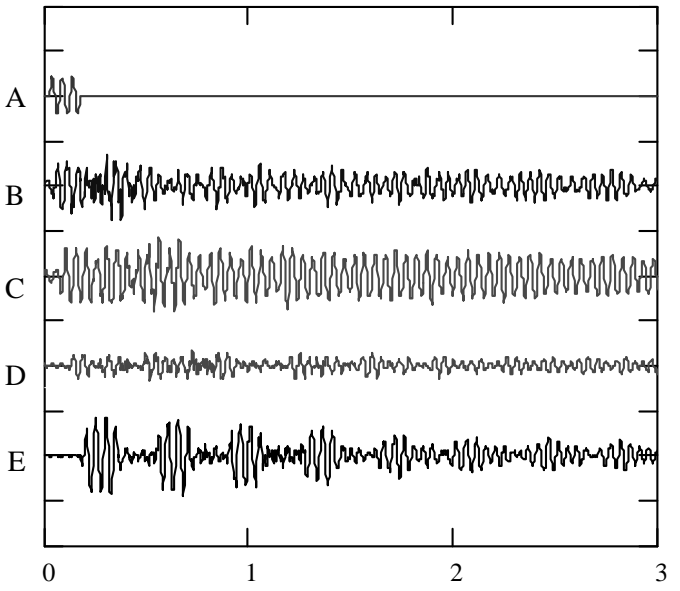

(c)

Time (ms)

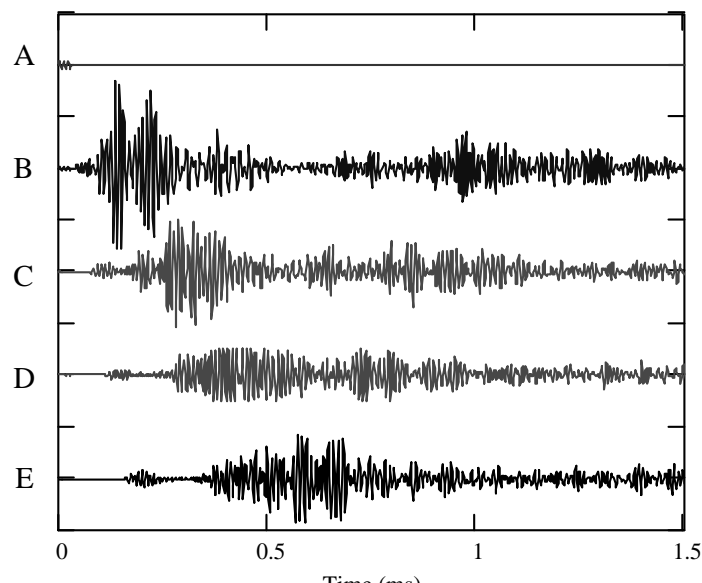

(b)

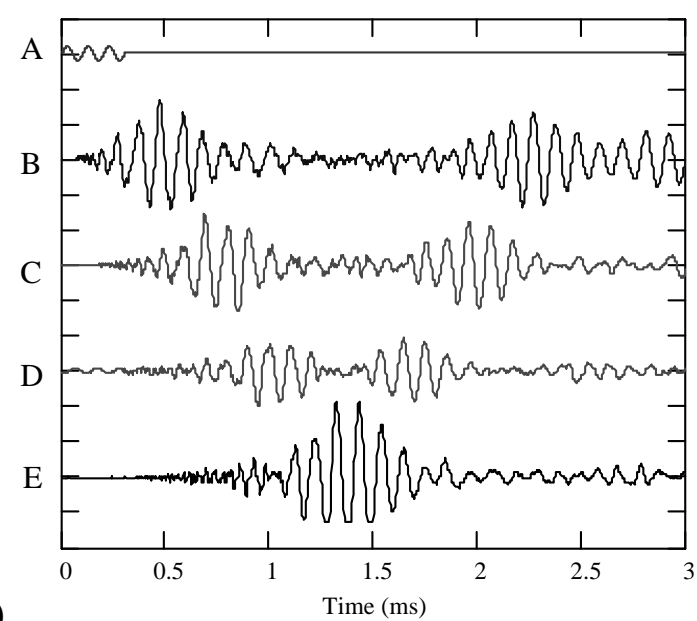

Fig. 13-Experimental data from the beam specimen: (a) $10 \mathrm{kHz}$ one-sided excitation, (b) $100 \mathrm{kHz}$ one-sided excitation, (c) $20 \mathrm{kHz}$ two-sided axial excitation, and (d) $10 \mathrm{kHz}$ two-sided flexural excitation

location A. It can be appreciated that two wave types (flexural and axial) are simultaneously excited. The flexural wave, which travels with lower speed, is excited more strongly than the axial wave. Nevertheless, minute examination of the signal, e.g., at sensor E, reveals that a small amplitude axial wave arrives there well ahead of the main flexural wave package. This figure also indicates how waves reflect at the beam boundary and travel backwards and forwards through the beam. Figure 13(b) shows the same phenomenon but at 100 $\mathrm{kHz}$. This time, the axial waves are much better distinguished as clear packets of smaller amplitude waves ahead of the main flexural waves. Because the axial waves are stronger at this frequency, they can be distinguished at all sensor locations, B-E. Figure 13(c) presents the result of applying a $20 \mathrm{kHz}$ symmetric excitation to the upper and lower active sensors existing at location A. Under symmetric excitation, only the axial waves are generated. Their speed is high, and they undergo multiple reflections from the ends of the beam. Figure 13(d) presents the result of applying a $10 \mathrm{kHz}$ antisymmetric excitation to the same active sensor pair at location A. This time, only the flexural waves are excited. They travel more slowly, but have much larger amplitude than the axial waves. Reflections from the beam ends and their traveling back and forth are apparent. From these plots, the time of flight for each wave package can be observed, recorded, and plotted against distance. Then, the group velocity of the wave package was calculated through linear regression.

\section{Frequency Tuning Effects}

During our experiments, we investigated the effect of excitation frequency on the excited wave amplitude. It was found that, at low frequencies (e.g., $10 \mathrm{kHz}$ ) the excitation of flexural waves was much stronger than that of axial waves. However, as frequency increases beyond $150 \mathrm{kHz}$, the excitation of flexural waves decreases, while that of axial waves increases significantly. A "sweet spot" for axial wave excitation was found in the 300-400 kHz range, as shown in Fig. 14(a). At $300 \mathrm{kHz}$, the first wave pack and the axial pack peaked. The second wave pack peaked at $350 \mathrm{kHz}$. We found that using clear-tone excitation a $300 \mathrm{kHz}$ gave very good and clean excitation of the $S_{0}$ waves. Since, at this frequency, the $\mathrm{S}_{0}$ waves have a shallow dispersion curve (cf. Fig. 3), the waves generated in this manner were observed to have very little dispersion. When moving away from this center frequency by about $\pm 25 \mathrm{kHz}$, the cleanness of this excitation 


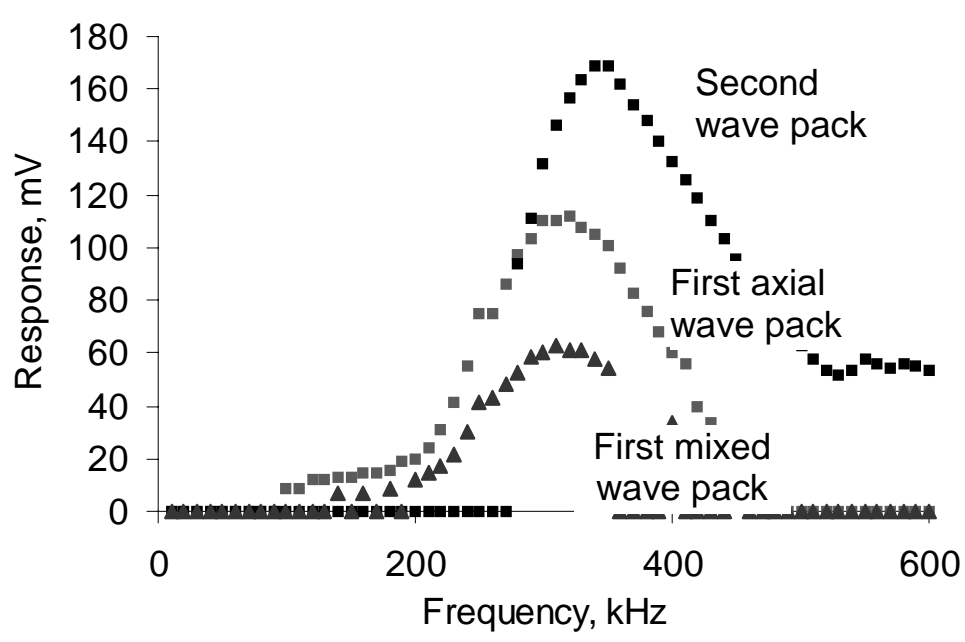

(a)

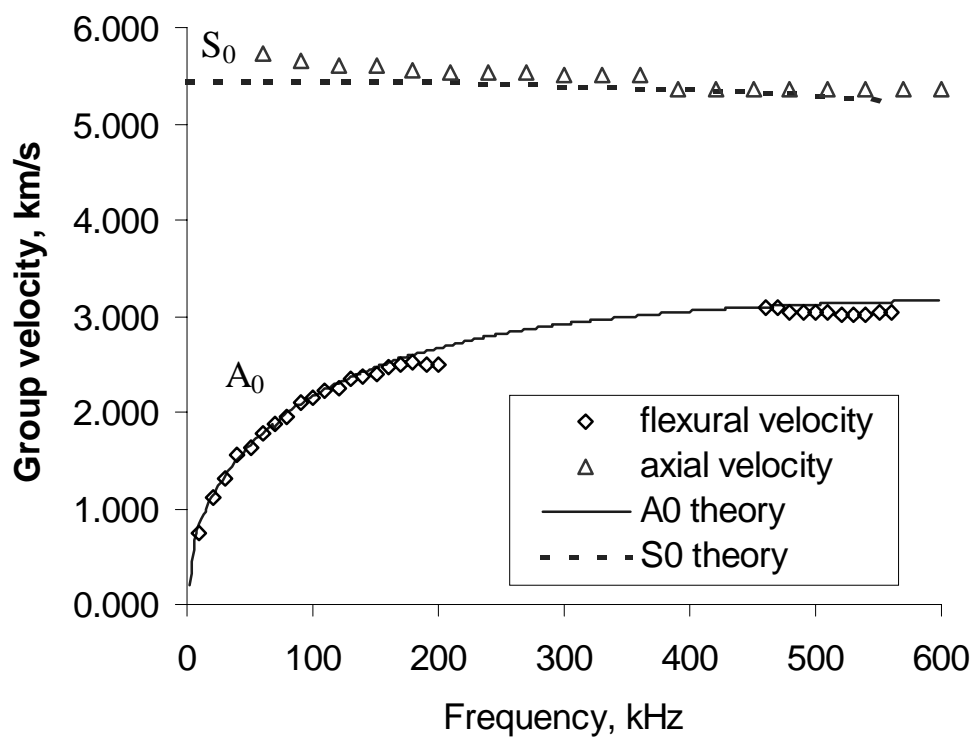

(b)

Fig. 14-Survey of Lamb wave excitation with a surface-mounted PWAS: (a) frequency tuning studies identified a maximum wave response around $300 \mathrm{kHz}$, and (b) group velocity dispersion curves

diminished because of $A_{0}$ waves were also excited. Since $\mathrm{A}_{0}$ waves are very dispersive in this frequency range (cf. Fig. 3), their excitation is to be avoided. Subsequently, for the plate experiments described in the next section, this excitation "sweet spot" at $300 \mathrm{kHz}$ was adopted as standard.

\section{Experimental Determination of Group Velocity Dispersion Curves}

Figure 14(b) shows a plot of the group velocity versus frequency, resulting from our experimental measurements. It shows the flexural wave data measured on the beam specimen, and the axial wave data measured on the plate specimen. Superposed on the same chart are the $A_{0}$ and $S_{0}$ group velocities curves predicted by Lamb wave theory. The concordance between the theoretical $\mathrm{A}_{0}$ group velocity and the measured $\mathrm{A}_{0}$ group velocity is remarkably good. The $\mathrm{A}_{0}$ data for the interval $200-450 \mathrm{kHz}$ was not measured because, in this interval, the flexural wave could not be excited due to the axial wave's dominance mentioned in the previous section (cf. Fig. 14(a)). The $\mathrm{S}_{0}$ data also show remarkably good concordance with the theoretical predictions, except at low frequencies $(f<100 \mathrm{kHz})$ where the excitation of axial waves is more difficult due to the flexural wave's dominance. Overall, the data presented in Fig. 14(b) indicate that the theoretically predicted Lamb wave group velocities have been experimentally confirmed.

\section{Main Findings of Beam Embedded Ultrasonics Investigation}

The main findings of investigating embedded ultrasonics on a $1 \mathrm{D}$ beam structure are as follows.

The modeling of wave propagation and reflection in thin strip beams was successfully achieved with the finite element method using shell elements and time integration. Both axial and flexural waves were separately studied. 


\begin{tabular}{lccccccccccc}
\hline & \multicolumn{10}{c}{ Sensor } \\
\cline { 2 - 12 }$y(\mathrm{~mm})$ & 1 & 2 & 3 & 4 & 5 & 6 & 7 & 8 & 9 & 10 \\
\hline$y(\mathrm{~mm})$ & 100 & 100 & 100 & 100 & 100 & 450 & 450 & 450 & 800 & 800 & 800 \\
& 175 & 250 & 325 & 400 & 100 & 250 & 400 & 100 & 250 & 400
\end{tabular}

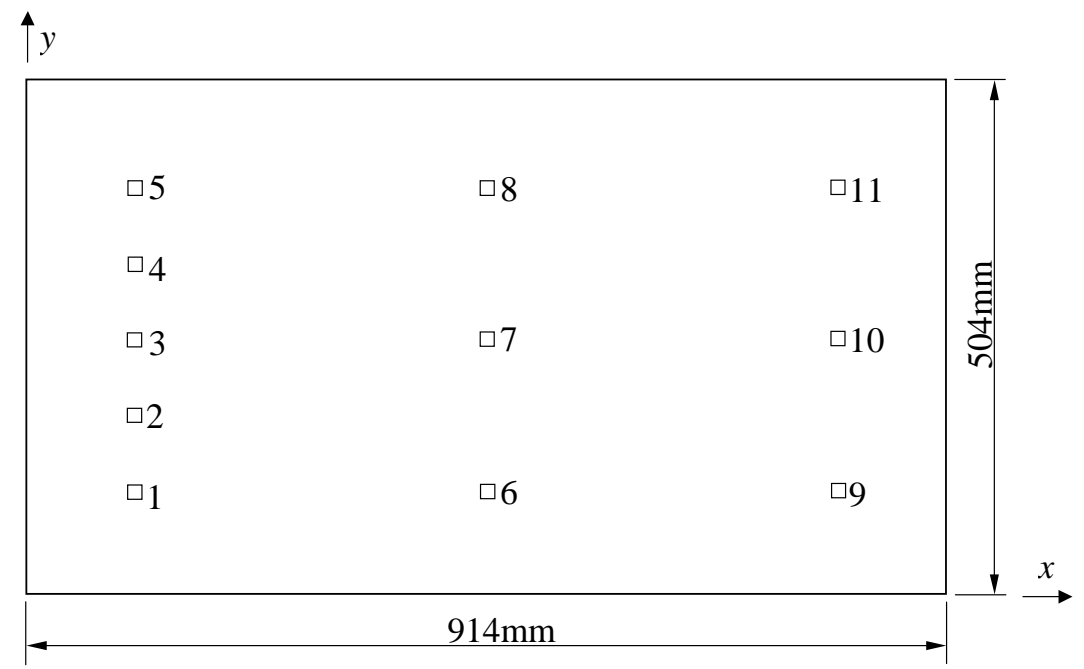

Fig. 15-Plate, $1.6 \mathrm{~mm}$ thick, 2024 aluminum alloy

The detection of internal cracks using the pulse-echo method with embedded ultrasonics hardware was successfully simulated at frequencies up to $100 \mathrm{kHz}$.

Embedded ultrasonics experiments were successfully performed on a thin strip beam specimen equipped with piezoelectric active sensors placed in pairs at five locations. The excitation and reception of both axial and flexural waves, separately and together, were verified.

Group velocity of the wave pack was successfully determined. The values were found to be within $5.6 \%$ of theoretical predictions, which is explainable in terms of manufacturer's tolerances in geometry and materials, as well as wave dispersion. The group velocity dispersion curves for flexural $\left(\mathrm{A}_{0}\right)$ and axial $\left(\mathrm{S}_{0}\right)$ Lamb wave modes were experimentally confirmed.

An excitation "sweet spot" at $300 \mathrm{kHz}$ was identified and adopted as standard for follow-up plate experiments, described next.

\section{Embedded Ultrasonics Experiments on Thin Plates}

Due to the high overhead of FEM wave simulation on 2D structures, this part of the study was performed only experimentally. A $1.6 \mathrm{~mm}$ thick, 2024-aluminum alloy plate (914 $\times 504 \times 1.6 \mathrm{~mm}^{3}$ ) was instrumented with an array of eleven $7 \times 7 \mathrm{~mm}^{2}$ PWASs. The $(x, y)$ locations of the eleven active sensors are given in Table 3, and illustrated in Fig. 15. The sensors were connected with thin insulated wires to a 16-channel signal bus that ended into two eight-pin connectors (Fig. 16). An HP33120A arbitrary signal generator was used to generate a $300 \mathrm{kHz}$ tone-burst excitation with a $10 \mathrm{~Hz}$ repetition rate. Both constant-amplitude and smoothed toneburst signals were used. The signal was sent at one active sensor that serves as a transmitter and received at the other ten active sensors that serve as receivers. Under the tone-burst excitation, the transmitter active sensor generated a package of elastic waves that spread out into the entire plate according to a circular wave-front pattern. A Tektronix TDS210 twochannel digital oscilloscope, synchronized with the signal generator, was used to collect the response signals from the active sensors. The two oscilloscope channels were digitally switched among the remaining ten active sensors using a digitally controlled switching unit. A data acquisition program was developed using LabView software to control the signal switch and record the data from the digital oscilloscope. In addition, a Motorola MC68HC11 microcontroller was tested as an embedded stand-alone controlling option.

Figure 17(a) shows the signals received at active sensors 1-10 when a constant-amplitude tone burst was applied at sensor 11 . The cleanness and consistency of the wave patterns are remarkable. In each signal, we notice a number of wave packs. The first of these packs corresponds to the wave received directly from the transmitter active sensor 11 . The subsequent wave packs correspond to waves reflected from the boundaries. The time of flight (TOF) of each wave pack is consistent with the traveled distance.

\section{Group Velocity Estimation}

These raw signals of Fig. 17(a) were processed using a narrow-band signal correlation algorithm followed by an envelope detection method. As a result, the exact TOF for each wave pack could be precisely identified. To perform a group velocity estimation, we used first the TOF of the first wave pack in each signal and plotted it against the geometric distance between the receiving active sensor and the transmitting active sensor (Fig. 17(b)). It can be appreciated from Fig. 17 (b) that a perfect straight line $\left(99.99 \% \mathrm{R}^{2}\right.$ correlation) 

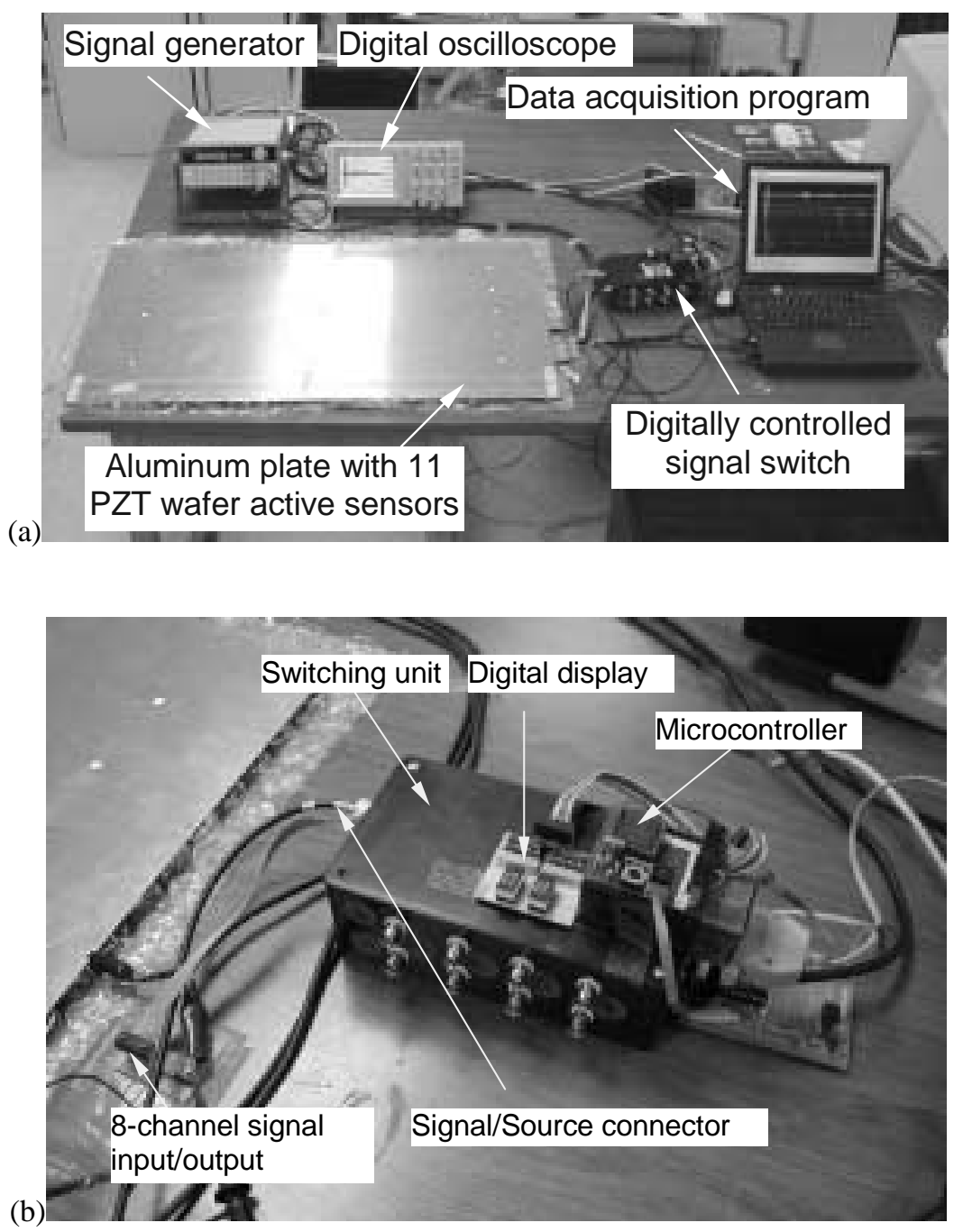

Fig. 16-Experimental setup for rectangular thin plate wave propagation experiment: (a) overall view showing the plate, active sensors, and instrumentation, and (b) detail of the microcontroller and switch box

TABLE 4-ELASTIC WAVE RECEPTION DATA ON THE THIN RECTANGULAR PLATE SPECIMEN

\begin{tabular}{ccccc}
\hline Sensor & $x(\mathrm{~mm})$ & $y(\mathrm{~mm})$ & $r(\mathrm{~mm})$ & $t(\mu \mathrm{s})$ \\
\hline 1 & 70 & 0 & 70.0 & 221 \\
2 & 84 & 0 & 84.0 & 263 \\
3 & 98 & 0 & 98.0 & 302 \\
4 & 112 & 0 & 112.0 & 357 \\
5 & 200 & 0 & 200.0 & 537 \\
6 & 200 & 96 & 221.8 & 602
\end{tabular}

was obtained. The slope of this line is the group velocity. Its value is $c_{g}=5.446 \mathrm{~km} \mathrm{~s}^{-1}$. For the $1.6 \mathrm{~mm}$ aluminum alloy used in this experiment, the theoretical $\mathrm{S}_{0}$-mode speed at $300 \mathrm{kHz}$ is $c_{S 0}=5.440 \mathrm{~km} \mathrm{~s}^{-1}$. Since, at this low frequency, the $\mathrm{S}_{0}$ mode has negligible dispersion, the group velocity and the wave speed have practically the same values. Hence, we compared the experimentally determined value of $5.446 \mathrm{~km}$ $\mathrm{s}^{-1}$ with the theoretical value of $5.440 \mathrm{~km} \mathrm{~s}^{-1}$. The speed detection accuracy $(0.1 \%)$ is remarkable. Based on the wave speed, we conclude that the first wave packets are $\mathrm{S}_{0} \mathrm{Lamb}$ waves.

\section{Reflections Analysis}

The next step in our analysis was to understand the reflection patterns, represented by the subsequent wave packs appearing in each signal. These packs, appearing after the first pack, represent waves that are reflected from the edges of the plate. Understanding the wave reflection patterns is essential for establishing the methodology for implementing the pulse-echo method for damage detection. In our analysis, we had to establish a correlation between the TOF of each wave pack and the distance traveled by that particular pack 


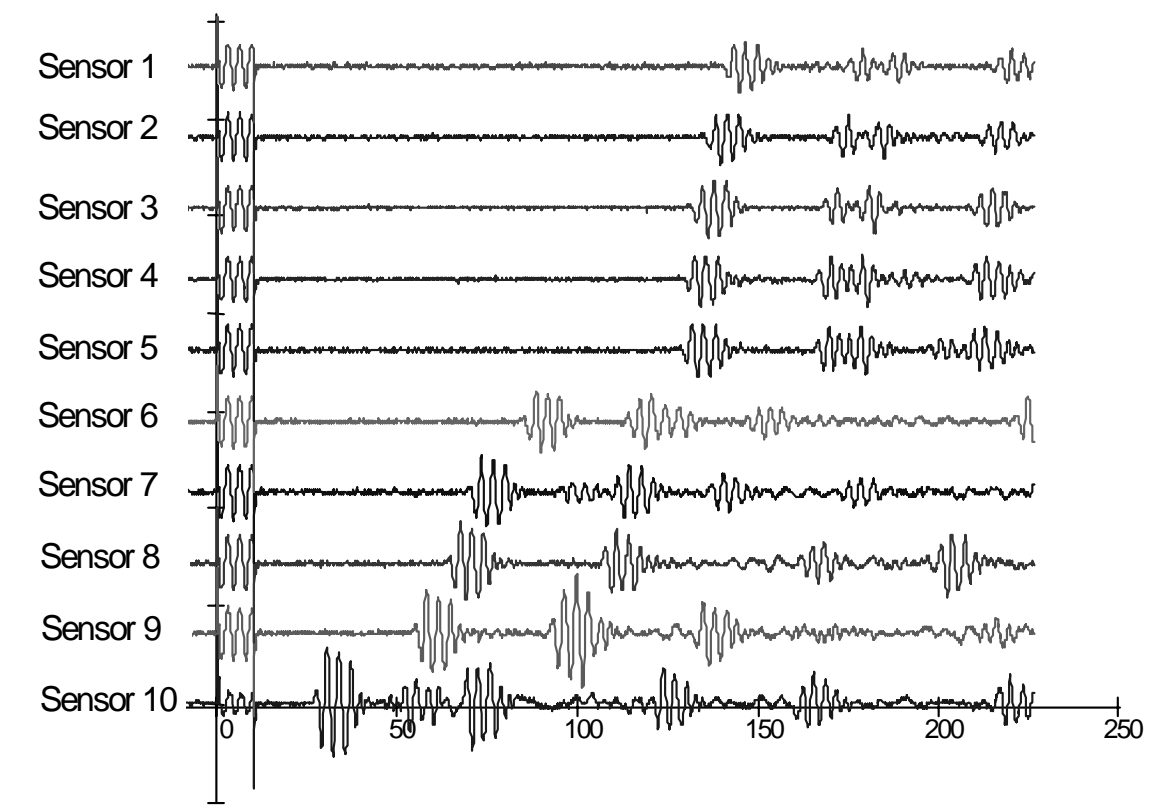

(a)

Time (micro-sec)

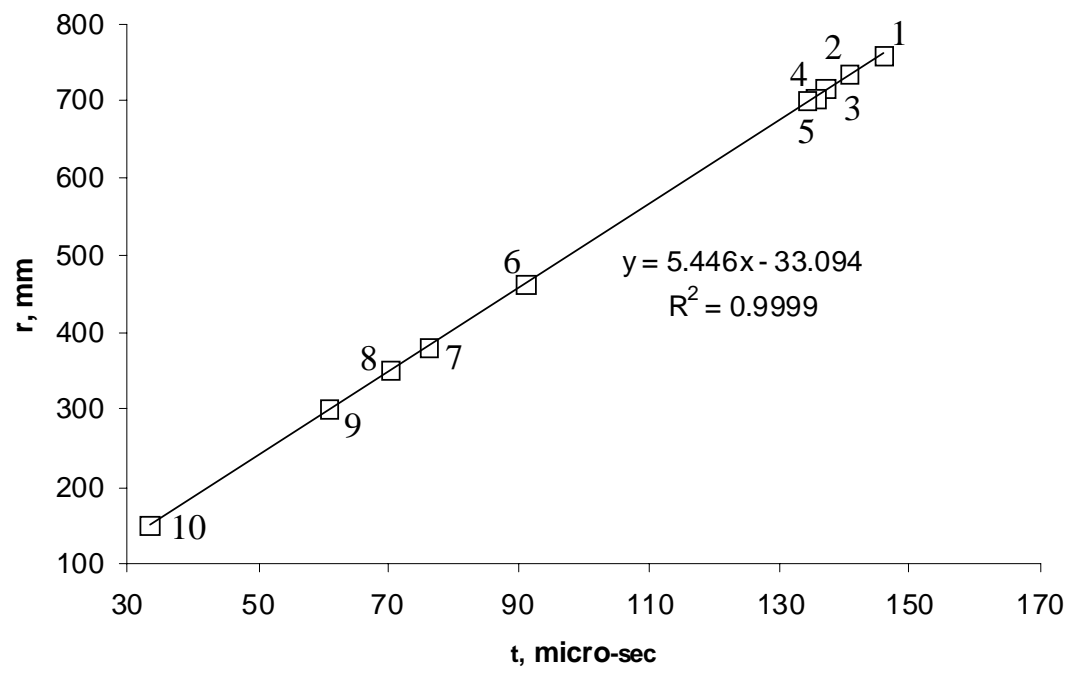

(b)

Fig. 17-(a) Reception signals received at active sensors 1-10 when a constant amplitude tone burst was applied at sensor 11 , and (b) Correlation between radial distance and TOF (numbers indicate sensor labels)

(path length). The TOF determination was immediate, but the determination of the actual traveled distance was more involved. Since elastic waves propagate in a circular wave front through the 2D plate, they reflect at all the edges and continue to travel around until fully attenuated. AutoCAD drawing software was used to assist the analysis and calculate the actual path length of the reflected waves. The drawing in Fig. 18(a) shows the plate and its mirror reflections with respect to the four edges and four corners. Thus, an image containing nine adjacent plates was obtained. To identify which reflection path corresponds to a particular wave pack, we took its TOF and multiplied it by the wave speed to obtain a first estimate of the path length. For example, Fig. 18(a) shows that for the reflected reception at sensor 6 , the estimated distance is $645 \mathrm{~mm}$. Then, a circle, centered at the transmitter sensor (11) and with its radius equal to the estimated distance, was drawn. The circle intersects with, or very close to, one of the reflected images of the reception sensor (6). In this way, we identified which of the reflected images of the reception sensor 6 as the actual image to be processed. Next, the true distance (path length) between the identified sensor image and the transmitter sensor was determined using the distance feature of the AutoCAD software. In Fig. 18(a), this true distance is $651.22 \mathrm{~mm}$. In this way, the path lengths for the reflection wave packs of all sensors were determined, and a TOF versus path length plot could be created (Fig. 18(b)). 


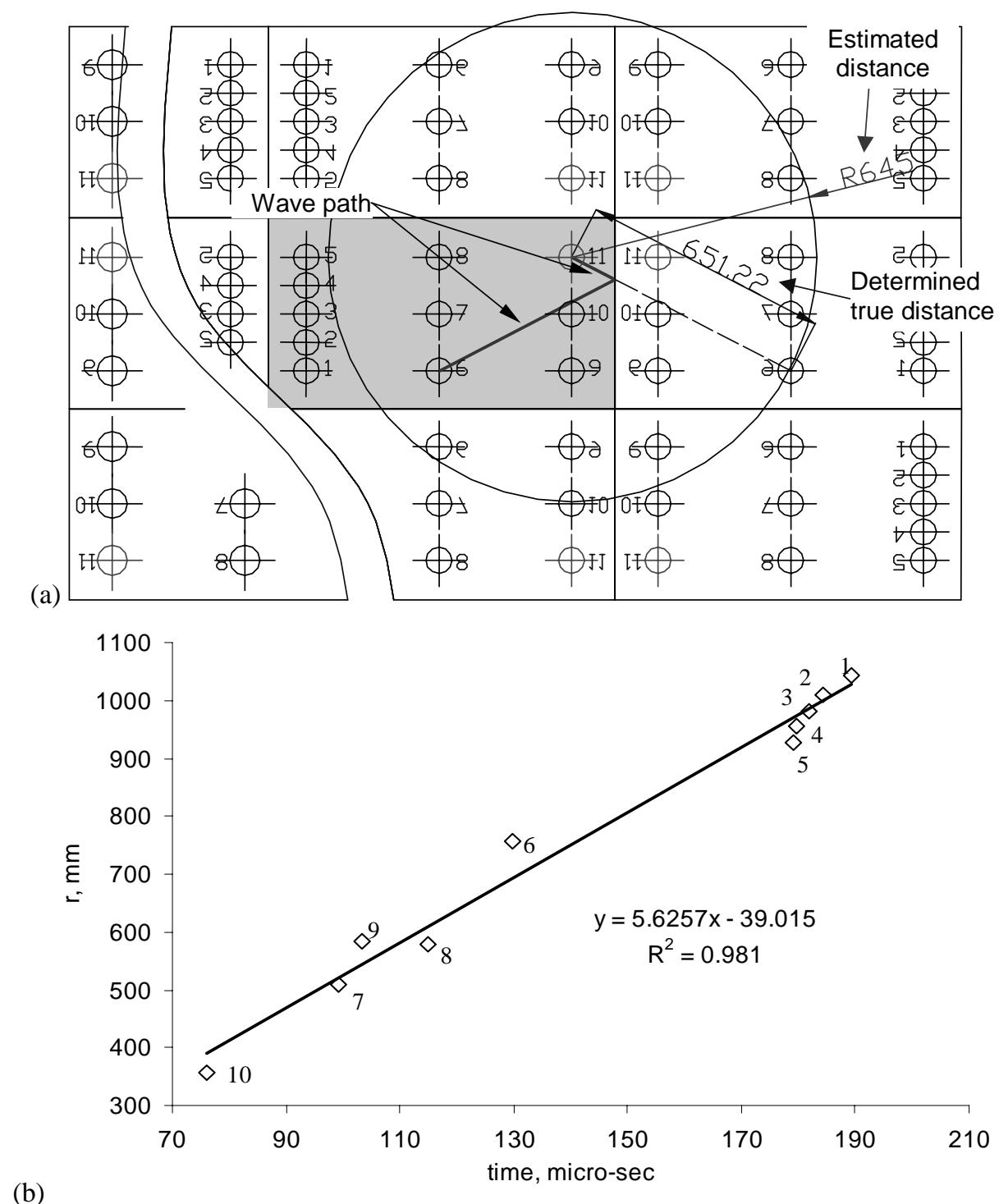

(b)

Fig. 18-Calculation of the distances traveled by the reflected waves: (a) the actual plate (shaded) surrounded by eight mirror images to assist in the calculation of the reflection path length, and (b) correlations between path length and TOF for the wave reflection signals (second wave pack) captured on active sensors 1-10

With the exception of one outlier, the TOF versus path length plot of Fig. 18(b) shows good linearity and thus proves the consistency of our reflection analysis.

\section{Pulse Echo Analysis}

In our plate experiments, we performed pulse-echo analysis on sensor 11. After the initial bang, the transmitter active sensor (11) was also able to capture subsequent signals representing waves reflected by the plate boundaries and sent back to the transmitter (Fig. 19). These subsequent wave packs were recorded for evaluating the pulse-echo method. Figure 19(a) shows the sensor 11 signal. This signal contains the excitation signal (initial bang), and a number of wave packs received in the pulse-echo mode. The wave generated by the initial bang undergoes multiple reflections from the plate edges, as shown in Fig. 19(b). The values of the true path length for these reflections are given in Table 6 . It should be noted that the path lengths for reflections $\mathrm{R}_{1}$ and $\mathrm{R}_{2}$ are very close. Hence, the echoes for these two reflections virtually superpose on the pulse-echo signal of Fig. 19(a). It is also important to notice that reflection $\mathrm{R}_{4}$ has two possible paths, $\mathrm{R}_{4 a}$ and $\mathrm{R}_{4 b}$. Both paths have the same length. Hence, the echoes corresponding to these two reflection paths arrive simultaneously and form a single echo signal in Fig. 19(a), with roughly double the intensity of the adjacent signals. Figure 19(c) shows the TOF of the echo wave packages plotted against their path lengths. The straight-line fit has a very good correlation $\left(\mathrm{R}^{2}=99.99 \%\right)$. The corresponding wave speed is $5.389 \mathrm{~km} \mathrm{~s}^{-1}$, i.e., within $1 \%$ of the theoretical value of $5.440 \mathrm{~km} \mathrm{~s}^{-1}$.

\section{Damage Detection Experiments}

Lamb-wave active-sensor damage detection strategy stems from the ultrasonic ${ }^{4}$ and acousto-ultrasonic ${ }^{6}$ damage 
(a)

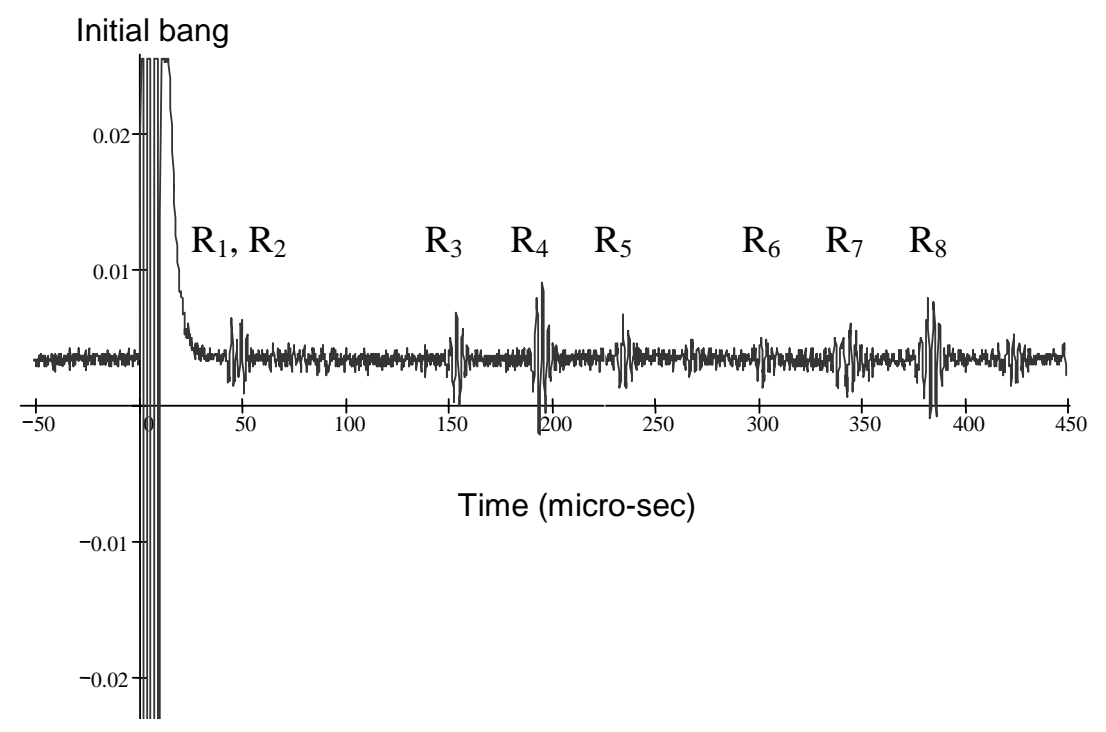

(b)
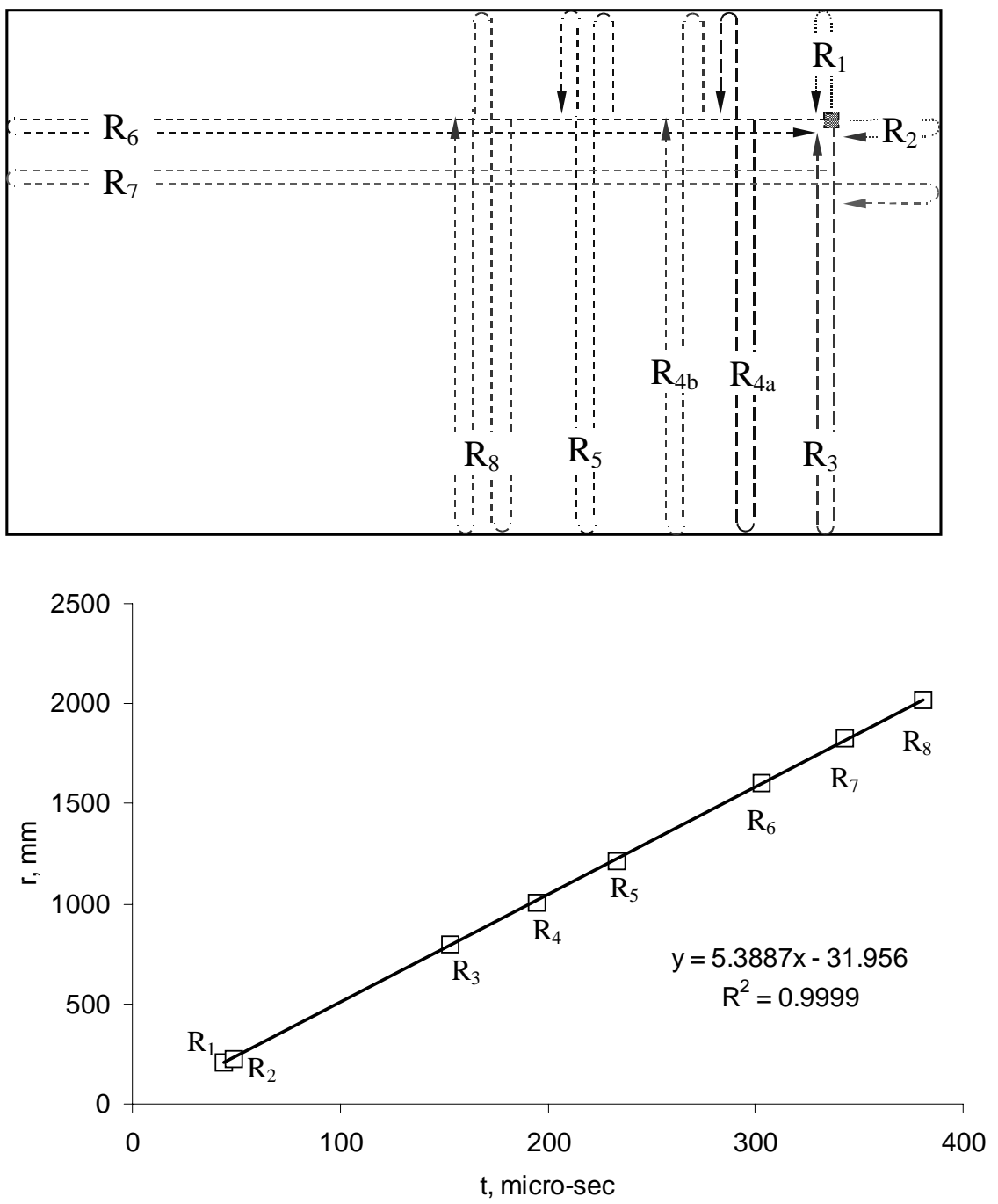

(c)

Fig. 19-Pulse-echo method applied to active sensor 11: (a) the excitation signal and the echo signals on active sensor 11, (b) schematic diagram of the wave paths for each wave pack, and (c) correlation between path length and TOF 
TABLE 5-ANALYSIS OF WAVE REFLECTION SIGNALS (SECOND WAVE PACK) CAPTURED ON ACTIVE SENSORS 1-10 ON RECTANGULAR PLATE SPECIMEN

\begin{tabular}{ccccccccccc}
\hline Active & & & & & & & & & \\
Sensor & 1 & 2 & 3 & 4 & 5 & 6 & 7 & 8 & 9 & 10 \\
\hline TOF $(\mu \mathrm{s})$ & 189.4 & 184.4 & 181.8 & 179.8 & 179.2 & 129.7 & 103.4 & 115 & 99.3 & 76.2 \\
Path length $(\mathrm{mm})$ & 1043.8 & 1009.7 & 980.2 & 955.6 & 928 & 755.8 & 583.6 & 578 & 508 & 358
\end{tabular}

\begin{tabular}{ccccccccc}
\multicolumn{7}{c}{ TABLE 6-ANALYSIS OF PULSE-ECHO SIGNALS OF SENSOR 11 ON RECTANGULAR PLATE SPECIMEN } \\
\hline Wave pack label & $\mathrm{R}_{1}$ & $\mathrm{R}_{2}$ & $\mathrm{R}_{3}$ & $\mathrm{R}_{4}$ & $\mathrm{R}_{5}$ & $\mathrm{R}_{6}$ & $\mathrm{R}_{7}$ & $\mathrm{R}_{8}$ \\
\hline TOF $(\mu \mathrm{s})$ & 43.8 & 48.8 & 152.8 & 194.4 & 233.2 & 302.8 & 343.2 & 380.8 \\
Path length $(\mathrm{mm})$ & 104 & 114 & 400 & 504 & 608 & 800 & 914 & 1008
\end{tabular}

detection methodologies. Damage detection experiments were conducted on a $1220 \mathrm{~mm}^{2}, 1 \mathrm{~mm}$ thick, aircraft-grade 2024-T3 aluminum panel. A $7 \times 7 \mathrm{~mm}^{2}, 0.2 \mathrm{~mm}$ thick piezoelectric wafer active sensor was adhesively mounted at the center of the panel. The pulse-echo method was used. A 10 V $300 \mathrm{kHz}$ three-count smoothed tone burst ("initial bang") was applied to the active sensor with a $10 \mathrm{~Hz}$ repetition rate (Fig. 20). Under this electric excitation, the active sensor, which is strain coupled with the plate surface, transmitted an elastic wave into the plate. The elastic wave traveled as a guided wave (Lamb wave $S_{0}$ mode) along the plate in an omnidirectional pattern. Upon reflection at the plate boundaries, the guided wave was reflected and received back as an echo. Consistent with the pulse-echo methods, the same active sensor was used for both transmission and reception. Figure 20(a) shows a TOF of $225.6 \mu$ s. Since the distance from the active sensor to the nearest edge is $610 \mathrm{~mm}$, and since the $\mathrm{S}_{0}$ mode travels, at this frequency, with a group velocity $c_{g}=5.440 \mathrm{~mm} \mathrm{us}^{-1}$, the theoretical round-trip TOF would be $\mathrm{TOF}_{\text {theory }}=224.3 \mu \mathrm{s}$. This compares very favorably with the experimental value of $225.6 \mu \mathrm{s}(0.6 \%$ error $)$. To illustrate crack detection, a $10 \mathrm{~mm}$ long, $0.127 \mathrm{~mm}$ wide simulated crack was manufactured at $305 \mathrm{~mm}$ from the sensor, and the pulse-echo methodology was repeated. The results of this test are shown in Fig. 20(b). An additional reflection is apparent at $\mathrm{TOF}_{\text {crack }}=113.0 \mu \mathrm{s}$. The theoretical round-trip TOF to the crack is $\mathrm{TOF}_{\text {crack-theory }}=112.1 \mu \mathrm{s}$. The accuracy of the crack detection method seems remarkable $(0.8 \%$ error).

\section{Main Findings of the Embedded Ultrasonics Investigation on a $2 D$ Plate}

The main findings of investigating embedded ultrasonics on a 2D plate structure are as follows.

Embedded ultrasonics experiments were successfully performed on a thin-gage rectangular plate specimen equipped with piezoelectric active sensors placed in an array pattern. The Lamb waves generated through this method were found to propagate omnidirectionally in a circular wave front over the entire plate surface.

Although excitation was possible at various frequencies, it was found that the $300 \mathrm{kHz}$ axial wave gave a more clear and distinct transmission and reception of the wave packs. The wave speed was successfully determined and the theoretical value was recovered with remarkable accuracy $(0.1 \%)$.

The wave signals obtained in the 2D plate were much cleaner than in the 1D narrow strip beam due to the lack of detrimental interference from the sides of the specimen.

Due to the low attenuation of these guided waves, multiple reflections were present and showed in the captured signal as distinct wave packs. These multiple reflections were analyzed and successfully interpreted.

The use of the pulse-echo method with the embedded PWAS was successfully demonstrated. A $19 \mathrm{~mm}$ hairline crack placed at $305 \mathrm{~mm}$ from the active sensor was detected with remarkable accuracy ( $0.8 \%$ error). This opened the way for developing an embedded ultrasonics damage detection methodology using an array of PWASs.

\section{Comparison Between PWASs and Conventional Ultrasonic Transducers}

PWASs are surface mounted transducers, intimately bonded to the material surface, which interact with the structure through stresses and strains parallel to the material surface, i.e., through a "pinching" action. In contrast, conventional ultrasonics transducers interact with the structure through forces and displacements normal to the material surface, i.e., through a "tapping" action. Both conventional ultrasonic transducers and PWASs can simultaneously act as transmitters and receivers of elastic waves. Conventional ultrasonic transducers cannot excite Lamb waves directly. To generate Lamb waves, they have to impinge on the plate obliquely through a coupling wedge and to use Snell's law to achieve the appropriate mode conversion. On the other hand, PWASs can excite Lamb and other guided waves directly, since their "pinching" action parallel to the material surface is natural to the guided wave excitation.

Conventional ultrasonic transducers are resonant devices that work optimally only at fixed frequencies. In contrast, PWASs are non-resonant transducers, which can cover a wide frequency range. Figure 21 shows PWAS calibration in the range $10-600 \mathrm{kHz}$; their use at up to $2.6 \mathrm{MHz}$ has been also demonstrated. ${ }^{23}$ Thus, a variety of guided waves can be generated with the same PWAS installation.

Conventional ultrasonic transducers generate guided plate waves only in a direction perpendicular to the wedge edge. ${ }^{31}$ In contrast, PWASs transmit and receive guided waves omnidirectionally.

PWASs are very low profile, unobtrusive, non-invasive, very low weight, and inexpensive. They can be embedded inside built-up structures. In contrast, conventional ultrasonic transducers are bulky, obtrusive, invasive, and much more expensive.

\section{Conclusions}

The main contribution of this paper lies in clarifying, through theoretical simulation and experimental measurements, the major aspects associated with using PWASs in 
(a)

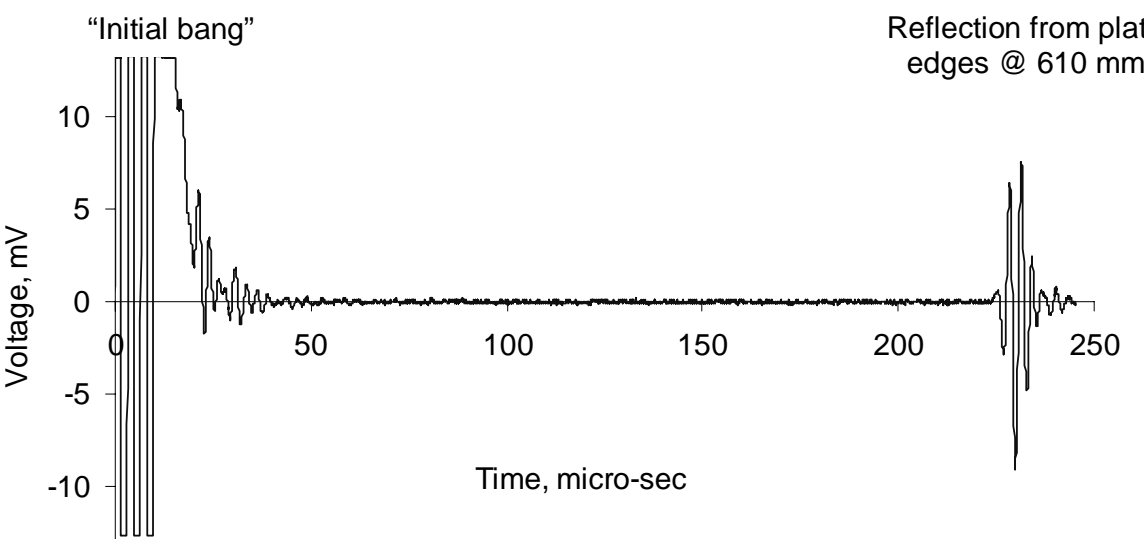

(b)

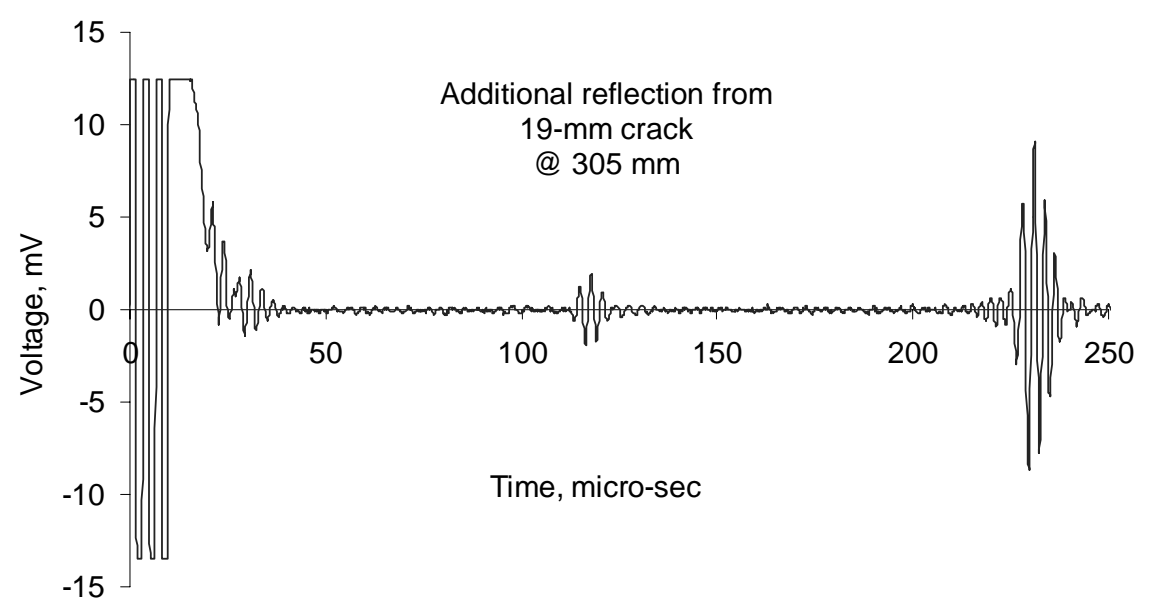

Fig. 20-Analysis of the pulse-echo active-sensor Lamb-wave crack detection experiment: (a) signal recorded on the pristine panel showing the reflection from the panel edges, and (b) signal recorded on the cracked panel showing, in addition, the reflection from the crack $\left(1220 \mathrm{~mm}^{2}, 1 \mathrm{~mm}\right.$ thick, 2024 T3 panel; $10 \mathrm{~mm}$ long, $0.127 \mathrm{~mm}$ wide simulated crack; $7 \mathrm{~mm}{ }^{2}, 0.2 \mathrm{~mm}$ thick PWAS)

conjunction with embedded ultrasonics damage detection and in situ structural health monitoring. The systematic theoretical and experimental investigations presented here have proved the following essential points.

The small $\left(7 \times 7 \times 0.2 \mathrm{~mm}^{3}\right)$ non-intrusive PWASs used in these experiments were capable of exciting axial and flexural Lamb waves that propagate in a circular wave front throughout a thin-gage plate structures. It is conceivable that similar propagation will also happen in thin-gage shells typical of aerospace structures.

The same active sensors were also capable of capturing the elastic wave signals, thus proving their dual functions. The captured signals were of remarkable strength (up to 50 $\mathrm{mVpp}$ ) and could be used directly without amplification or other signal conditioning.

The experimental studies were supported by extensive finite element analysis that permitted the simulation of axial and flexural waves in specimens in pristine conditions as well as with internal cracks of various sizes.

An excitation "sweet spot" for $\mathrm{S}_{0}$ waves was discovered at $300 \mathrm{kHz}$ in $1 \mathrm{~mm}$ aluminum plates. This PWAS mode- tuning phenomenon, not yet reported by other investigators, was essential in the success of our experiments.

The pulse-echo technique, which is essential for damage detection with ultrasonic methodology, was successfully demonstrated in both beam and plate studies. In beam studies, the pulse-echo technique was demonstrated using FEM simulation of wave propagation in a specimen with a central crack of various sizes. In plate studies, the pulse-echo method was demonstrated by identifying the trail of echoes resulting from the wave being reflected from various plate boundaries.

The waves generated during this experiments showed remarkable clarity and could be easily interpreted. The experiments displayed a very good correlation between the TOF and the wave path length that permitted the determination of the wave speed (group velocity) with up to $99.9 \%$ accuracy. This high accuracy will ensure accurate detection of internal defects when the method is applied in structural health monitoring.

This theoretical and experimental study of elastic wave generation and reception has confirmed beyond any doubt that PWASs could be used to (a) generate true ultrasonic 
wave in thin-gage metallic structures, and (b) simultaneously to collect the wave propagation signals. The "true" nature of the generated ultrasonic waves lies in their high frequency $(300 \mathrm{kHz})$, while previous studies published in the literature did not go beyond $10-20 \mathrm{kHz}$. The use of true ultrasonic frequencies allowed us to use the pulse-echo method with remarkable success.

The results reported in this paper open the path for applying the well-established theories and methodologies of ultrasonic testing to this new emerging field of embedded ultrasonics. The use of small and inexpensive PWASs instead of the conventional costly and bulky ultrasonic transducers opens up the opportunity for creating arrays of embedded ultrasonic active sensors that can non-intrusively perform in situ structural health monitoring. Although the active-sensor experiments reported here were conducted only on thin metallic plates of simple geometries, the findings of this study can be easily extended to practical geometries and to composite material structures.

\section{Acknowledgments}

The authors gratefully acknowledge partial support from the Department of Energy through the Sandia National Laboratories contract BF 0133, from the National Science Foundation grants NSF CMS-9908293 and NSF INT-9904493, and the participation in the U.S. Air Force Summer Faculty Fellowship Program 2002.

\section{References}

1. Krautkramer, J. and Krautkramer, H., Ultrasonic Testing of Materials, Springer-Verlag, Berlin (1990).

2. Blitz, J. and Simpson, G., Ultrasonic Methods of Non-Destructive Testing, Chapman and Hall, London (1996).

3. Cawley, P., "Quick Inspection of Large Structures Using Low Frequency Ultrasound,” Structural Health Monitoring-Current Status and Perspective, Fu-Kuo Chang, editor, Technomic, Inc. (1997).

4. Rose, J.L., Ultrasonic Waves in Solid Media, Cambridge University Press, Cambridge (1999).

5. Rose, J.L., "NDEApplications of Guided Waves," 7th Non-Destructive Evaluation Topical Conference, ASME International, April 23-25, San Antonio, $T X$ (2001).

6. Duke, J.C. Jr., Acousto-Ultrasonics-Theory and Applications, Plenum, New York (1988).

7. Crawley, E.F. and Lazarus, K.B., "Induced Strain Actuation of Isotropic and Anisotropic Plates,” AIAA Journal, 29 (6), $944-951$ (1991).

8.Tzou, H.S. and Tseng, C.I., "Distributed Piezoelectric Sensor/Actuator Design for Dynamic Measurement/Control of Distributed Parametric Systems: A Piezoelectric Finite Element Approach,” Journal of Sound and Vibration, 138, 17-34 (1990).

9. Dimitriadis, E.K., Fuller, C.R. and Rogers, C.A., "Piezoelectric Actuators for Distributed Vibration Excitation of Thin Plates,” Journal of Vibration and Acoustics, 113, 100-107 (1991).

10. Lester, H.C. and Lefebvre, S., "Piezoelectric Actuator Models for Active Sound and Vibration Control of Cylinders," Journal of Smart Materials and Structures, 4, 295-306 (1993).

11. Song, O. and Librescu, L., "Vibration and Instability of Spinning Thin-Walled Beams Featuring Anisotropy and Pretwist, and Incorporating Adaptive Capabilities," Proceedings of the SPIE 5th International Symposium on Smart Structures and Materials, March 1-5, Catamaran Resort Hotel, San Diego, CA, paper 3323-49 (1998).
12. Saravanos, D.A. and Heyliger, P.R., "Coupled Layerwise Analysis of Composite Beams with Embedded Piezoelectric Sensors and Actuators," Journal of Intelligent Material Systems and Structures, 6 (3), 350-363 (1995).

13. Keilers, C.H. and Chang, F.-K., "Identifying Delamination in Composite Beams Using Built-in Piezoelectrics: Part I-Experiments and Analysis; Part II-An Identification Method," Journal of Intelligent Material Systems and Structures, 6, 649-672 (1995).

14. Chang, F.-K., "Manufacturing and Design of Built-in Diagnostics for Composite Structures," 52nd Meeting of the Society for Machinery Failure Prevention Technology, Virginia Beach, VA, March 30-April 3 (1998).

15. Chang, F.-K., "Structural Health Monitoring: Aerospace Assessment," AeroMat 2001, 12th ASM Annual Advanced Aerospace Materials and Processes Conference, 12-13 June, Long Beach, CA (2001).

16. Moetakef, M.A., Joshi, S.P. and Lawrence, K.L., "Elastic Wave Generation by Piezoceramic Patches,” AIAA Journal, 34 (10), 2110-2117 (1996).

17. Lakshmanan, K.A. and Pines, D.J., “Modeling Damage in Composite Rotorcraft Flexbeams Using Wave Mechanics,” Journal of Smart Materials and Structures, 6 (3), 383-392 (1997).

18. Blanas, P., Wenger, M.P., Shuford, R.J. and Das-Gupta, D.K., "Active Composite Materials and Damage Monitoring," Proceedings of the International Workshop on Structural Health Monitoring, Stanford University, CA, September 18-20, 199-207 (1997).

19. Blanas, P., Wenger, M.P., Rigas, E.J. and Das-Gupta, D.K., “Active Composite Materials as Sensing Element for Fiber Reinforced Smart Composite Structures," Proceedings of the SPIE North American Conference on Smart Structures and Materials, Proceedings of the SPIE 3329, San Diego, CA, March 1-5 (1998).

20. Lichtenwalner, P.F., "Local Area Damage Detection in Composite Structures Using Piezoelectrics,” Proceedings of Smart Structures and Materials, Santiago, CA, March 1-6 (1998).

21. Jiang, Z., Kabeya, K. and Chonan, S., "Longitudinal Wave Propagation Measuring Technique for Structural Health Monitoring," Proceedings of the SPIE Conference on Smart Structures and Integrated Systems, Newport Beach, CA, March (1999).

22. Osmont, D., Devillers, D. and Taillade, F., “A Piezoelectric Based Health Monitoring System for Sandwich Plates Submitted to Damaging Impacts," European Congress of Computational Methods in Applied Sciences and Engineering ECCOMAS 2000, Barcelona, September 11-14 (2000).

23. Giurgiutiu, V., Redmond, J., Roach, D. and Rackow, K., “Active Sensors for Health Monitoring of Aging Aerospace Structures,” 7th SPIE Annual International Symposium on Smart Structures and Materials, 5-9 March, Newport Beach, CA, paper 3985-103, Vol. 3985, 294-305 (2000).

24. Giurgiutiu, V. and Zagrai, A., "Embedded Self-Sensing Piezoelectric Active Sensors for On-Line Structural Identification," ASME Journal of Vibration and Acoustics, 124, 116-125 (2002).

25. Bartkowicz, T.J., Kim, H.M., Zimmerman, D.C. and Weaver-Smith, S., "Autonomous Structural Health Monitoring System: A Demonstration," Proceedings of the 37th AIAA/ASME/ASCE/AHS/ASC Structures, Structural Dynamics, and Materials Conference, Salt Lake City, UT, April 15-17 (1996).

26. Boller, C., Biemans, C., Staszewski, W., Worden, K. and Tomlinson, G., "Structural Damage Monitoring Based on an Actuator-Sensor System," Proceedings of SPIE Smart Structures and Integrated Systems Conference, Newport, CA, March 1-4 (1999).

27. ANSI/IEEE Standard 176, IEEE Standard on Piezoelectricity, The Institute of Electrical and Electronics Engineers, Inc. (1987).

28. Kolsky, H., "Stress Waves in Solids," Dover Publications, New York (1963).

29. Viktorov, I.A., Rayleigh and Lamb Waves-Physical Theory and Applications, Plenum Press, New York (1967).

30. Graff, F.K., "Wave Motion in Elastic Solids," Oxford University Press, Oxford (1975).

31. Deutsch, W.A.K, Cheng, A. and Achenbach, J.D., "Focusing of Rayleigh Waves: Simulation and Experiment," IEEE Transactions of Ultrasonics, Ferroelectrics, and Frequency Control, 46 (2), 333-340 (1999). 